\title{
Diversity of Chrysomelidae (Coleoptera) in Galicia, Northwest Spain: estimating the completeness of the regional inventory
}

\author{
ANDRÉS BASELGA* and FRANCISCO NOVOA \\ Departamento de Biología Animal, Facultad de Biología, Universidad de Santiago de Compostela, \\ 15706 Santiago de Compostela, Spain; *Author for correspondence: Address: Museo Nac. de Ciencias \\ Naturales, Dept. de Biodiversidad y Biología Evolutiva, c/José Gutiérrez Abascal, 2, 28006 Madrid, \\ Spain (e-mail: baselga@usc.es)
}

Received 10 June 2004; accepted in revised form 11 November 2004

Key words: Chrysomelidae, Cumulative models, Galicia, Iberian peninsula, Inventory, Species richness

\begin{abstract}
The diversity of Chrysomelidae (Coleoptera) in Galicia, Northwest Spain was examined. A long-term sampling was conducted during 1996-2001 and 267 species were collected, but including bibliographic citations a total of 276 species were recorded. As a result of this study the regional inventory has grown from 83 taxa cited before 1998 to the current 276 species. Species accumulation models were used to measure the inventory completeness and estimate the actual species richness of Chrysomelidae occurring in Galicia. Estimates were generated by analyzing both the rarefaction curve from the long-term sampling and the cumulative number of species recorded from Galicia since 1866. Values of total richness predicted by these different methods range between 290 and 323 species. Therefore, it seems that between 85 and $95 \%$ of the leaf beetle fauna was recorded and thus the inventory has reached an acceptable level of completeness.
\end{abstract}

\section{Introduction}

The family Chrysomelidae is a highly diverse phytophagous group which represents an important proportion of the Coleoptera, and thus the whole diversity of terrestrial communities. Estimations for Iberian and world Coleoptera indicate that leaf beetles reach about $10 \%$ of species within the order (Martin-Piera and Lobo 2000). At local scale the same pattern was observed in Galicia, where Chrysomelidae represent about $15 \%$ of the recorded beetles in Natural Park of Fragas del Eume (Baselga and Novoa 2004), though this percentage should be slightly reduced if some incompletely studied families were added to the inventory. Therefore, leaf beetle inventories and reliable estimations of its completeness are interesting tools for assessing biodiversity patterns, and thus optimize the conservation effort.

With this purpose, many papers were focused on the need to determine the degree of completion of faunistic inventories and thus estimate the true species richness for a wide range of taxonomic groups. Two different methods can be applied to this problem. The use of randomized sample accumulation curves was firstly developed for standardized samplings (Soberón and Llorente 1993; 
Colwell and Coddington 1994; Carlton and Robison 1998; Moreno and Halffter 2000; Summerville et al. 2001; Noguera et al. 2002) but also was applied to non-standardized data from museum collections or taxonomic databases (Soberón et al. 2000; Hortal et al. 2001, 2004; Petersen et al. 2003; Martín-Piera and Lobo 2003; Meier and Dikow 2004). A second way to determine de degree of completion of species inventories in a region is the growth over time of the cumulative species number as a function of the year of description (Medellín and Soberón 1999; Cabrero-Sañudo and Lobo 2003).

Knowledge about the Iberian leaf beetle diversity has been notably increased since last quarter of 20th century, with many papers dealing on faunistics of mountain areas (Daccordi and Petitpierre 1977; Petitpierre 1981, 1994, 1997; Petitpierre and Gómez-Zurita 1998; García-Ocejo et al. 1992; García-Ocejo and Gurrea 1995) or greater regions (Petitpierre 1980, 1983, 1988, 1999, 2000; Biondi 1991; Bastazo et al. 1993; Doguet et al. 1996) Most of these studies are focused on Mediterranean regions that occupy the greatest part of the Iberian peninsula, whereas Eurosiberian areas located in northern regions are still poorly known. This was the case of Galicia, northwest Spain (Figure 1), with only 83 species cited before 1998 (most of them recorded by López Seoane 1866; Heyden 1870; Chapman and Champion 1907; Iglesias 1928). Galicia is mostly located within Eurosiberian region, but some southeastern areas are included in Mediterranean region (Izco 1987; Rivas-Martínez 1987).

We carried out an extensive study of Chrysomelidae fauna from Galicia and some faunistic and taxonomic results were partially published (see Appendix A for references). The purpose of this paper is (i) to describe the regional species richness of this highly diverse group, and (ii) to estimate the completeness of

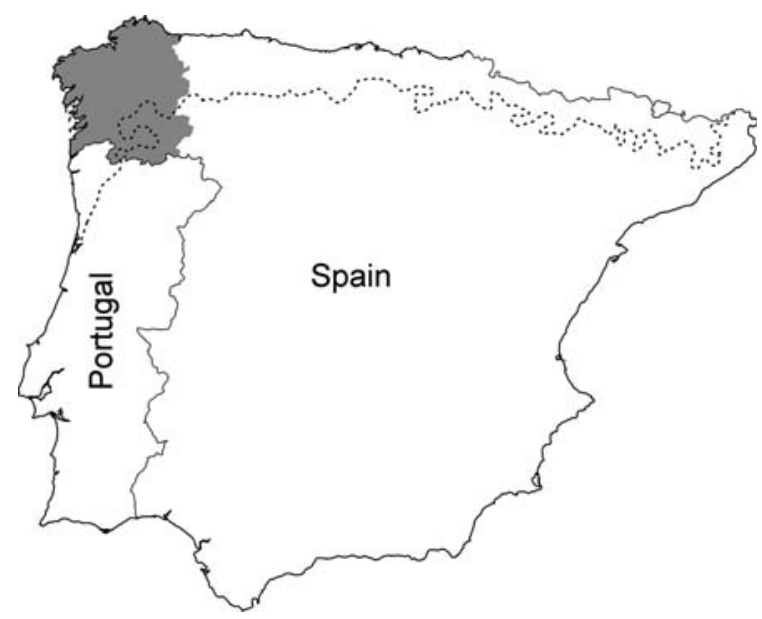

Figure 1. Location of the studied area in the northwest of the Iberian peninsula. Galicia (shadowed) is located in the boundary between Eurosiberian and Mediterranean phytogeographic regions (discontinuous line). 
the inventory, assessing both the rarefaction curve generated from one longterm sampling (1996-2001) which was the first attempt to get a significant picture of the regional leaf beetle fauna, and the cumulative number of species recorded since the first citations in 1866.

\section{Materials and methods}

Long-term sampling was carried out by Baselga, between 1996 and 2001, studying 191 localities in Galicia that were visited at least once (Figure 2). Among them, eight areas were selected and intensively sampled: coastal dunes and associated marshes (A), agricultural landscape near Santiago de Compostela (B), low altitude Atlantic mixed forest in Fragas del Eume Natural Park (C), medium altitude mountain ranges of Dorsal Gallega (D) and Larouco $(\mathrm{E})$, the temperate valley of the Sil river $(\mathrm{G})$ and high mountain ranges of Ancares $(\mathrm{F})$ and Eixo-Segundera $(\mathrm{H})$. Other localities visited during the field study are noted as $\mathrm{X}$ in Appendix A, whereas locations extracted from bibliographic sources are noted as $\mathrm{Y}$. Areas $\mathrm{E}, \mathrm{G}$ and $\mathrm{H}$ are located in the Mediterranean phytogeographic region, all the others in the Eurosiberian region (Izco 1987; Rivas-Martínez 1987). A total of 8602 specimens were collected. Another important source of material was the sampling carried out by G. Cerviño (1190 specimens) between 1991 and 1994. All this material is

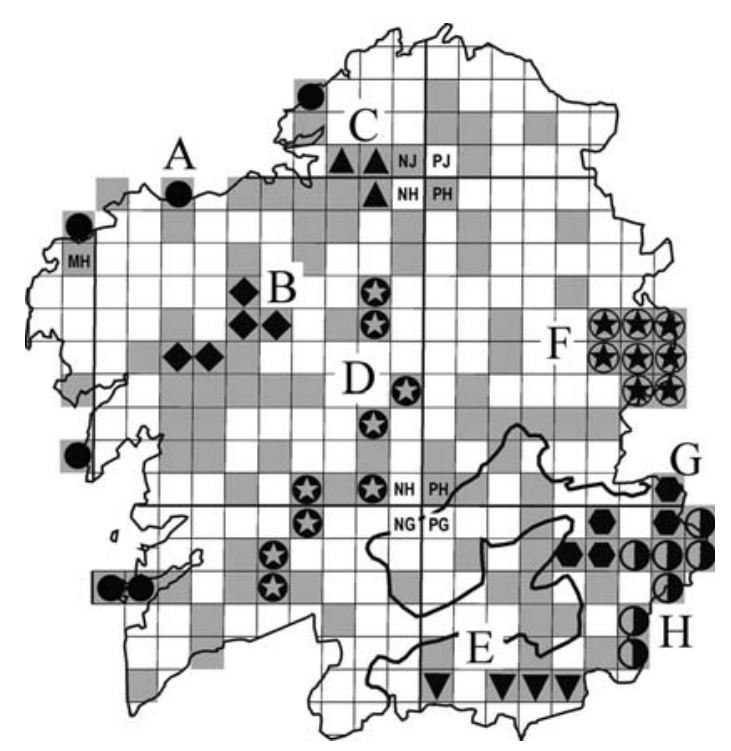

Figure 2. Sampling localities and selected areas noted in Appendix A. The squared pattern corresponds to the $10 \mathrm{~km}$ UTM grid. Galicia is located within $100 \mathrm{~km}$ squares MH, NG, NH, NJ, PG, $\mathrm{PH}$ and PJ. Sampled squares are shadowed and intensively sampled areas are noted with different symbols and respective letters. See Materials and methods for further explanation. 
deposited in Baselga collection in the Departamento de Biología Animal, Universidad de Santiago de Compostela, Spain.

To assess the completeness of the inventory both asymptotic model and nonparametric estimators (ICE, Chao 2 and jackknife of first order) were used (Colwell and Coddington 1994). Estimations were produced by two different approaches: (i) the rarefaction curve from long-term sampling and (ii) the cumulative number of recorded species since the first citations (López Seoane 1866) to the present.

The rarefaction curve and the non-parametric estimators were generated with EstimateS 6.0 software (Colwell 2000), randomizing the sample order 100 times. Database records were used as a sampling-effort surrogate (Soberón et al. 2000; Hortal et al. 2001; Martin-Piera and Lobo 2003). Our database comprised 3332 records for 10098 specimens and includes the results of the collections by G. Cerviño (1991-1994) and Baselga (1996-2001), along with some other specimens collected by Novoa and collaborators since 1973. Each record is comprised of the following fields: species name, locality, date, host plant, number of specimens and collector. Any difference in any database field value give rise to a new database record, so increments of the number of records provide correlative increments of the sampling effort (Martin-Piera and Lobo 2003). Thereafter, the asymptotic Clench function was fitted to the smoothed curve (Soberón and Llorente 1993; Hortal et al. 2004):

$$
S_{(e)}=a e /(1+b e)
$$

where $S_{(e)}$ is the number of species found per sampling-effort unit (e); $a$ and $b$, the parameters of the function. The later were adjusted to the data of each curve by means of a Simplex and Quasi Newton method (StatSoft 2001). The predicted asymptote is calculated as $a / b$.

The second estimation to determine the degree of completeness of Galician leaf beetle inventory was produced by fitting the Clench function to the cumulative number of recorded species since the first citations in the region (López Seoane 1866) to the present, following Cabrero-Sañudo and Lobo (2003) but considering the first Galician record instead of the year of description. This historic curve was generated taking into account the year of published papers or the year of collection if known. The final section (19912001) of this curve seems to get an asymptotic shape due to the increase in sampling effort and thus only this period was selected to adjust the Clench function. We considered the case of the complete sampling with two different collectors (1991-2001) and the case of the final period with an single collector (1996-2001). In the first case the shape of the curve is more irregular due to the lack of sampling effort in 1994 and 1995 but, on the other hand, it is used a higher number of points to estimate the function than in the second case. Therefore, both estimates (and the contrast between them) are interesting for different reasons.

Biogeographic patterns were synthesized following the chorotypes proposed by Vigna Taglianti et al. (1992) and then grouping them into four major 
categories: Iberian elements (Ibe), for species endemic from the Iberian Peninsula; Mediterranean elements (Med), for species widespread in the Mediterranean countries; Eurosiberian elements (Eur), for species widespread in Europe, or Europe and the Siberian range; and finally the wide range elements (WR), for species widespread in all or a great part of the Palaearctic region, and reaching parts of both Eurosiberian and Mediterranean areas. These major divisions are established in order to make clear the contrast between Eurosiberian (septentrional) and Mediterranean (meridional) contributions to Galician fauna which is located across the Eurosiberian-Mediterranean boundary. The other two categories are neutral regarding this aspect, because almost all Iberian elements are present in both sides of Eurosiberian-Mediterranean limit, as well as WR elements reach both regions.

\section{Results}

A total of 276 species have been recorded from Galicia, including the bibliographic citations (Appendix A) and 267 of them were recorded during the field study. The complete inventory (276 taxa) represents between 34\% (Petitpierre 2000) and 44\% (Vela and Bastazo 1999) of the total Iberian Chrysomelidae diversity, since there are two different estimations of actual number of Iberian leaf beetles. The Galician fauna of leaf beetles is comprised of about the same proportion of Eurosiberian (Eur: 19.2\%) and Mediterranean (Med: 19.6\%) elements. Species of wide range (WR) reach near half of the fauna $(45.7 \%)$ and Iberian endemisms (Ibe) represent the $15.2 \%$.

Two new species were described from Galicia: Aphthona sandrae (Baselga and Novoa 2002a) and Psylliodes cervinoi (Baselga and Novoa 2003) which are only known from their type localities. Eight taxa were recorded from the Iberian peninsula for the first time: Oulema erichsonii (Suffrian), Phyllotreta exclamationis (Thunberg), Phyllotreta ganglbaueri Heikertinger, Longitarsus australis (Mulsant and Rey), Longitarsus fulgens (Foudras), Chaetocnema confusa (Boheman), Psylliodes vindobonensis Heikertinger and Cassida subreticulata Suffrian (Baselga and Novoa 1998, 1999a, b, 2000c, 2001a, b, 2002a, b). Taking into account present new records ( 22 species) and previous papers, 193 species (69.9\% of known richness) have been newly recorded for Galicia since 1998.

The species accumulation curve (Figure 3) generated from the field study (excluding bibliographic records) nearly reaches the asymptote (292) predicted by the Clench function. Moreover, the observed richness $(S=267)$ is not far from the non-parametric estimators ICE (297), Chao 2 (300) and Jackknife 1 (311). Therefore between 85.9 and $91.4 \%$ of the estimated number of species living in Galicia seems to be detected in our field study (Table 1), but we should expect that between 24 and 44 taxa will be added to the inventory in the future.

On the other hand, the cumulative number of species recorded per year (Figure 4a) shows a slow growth since 1866 to 1990 and thereafter a great rise 


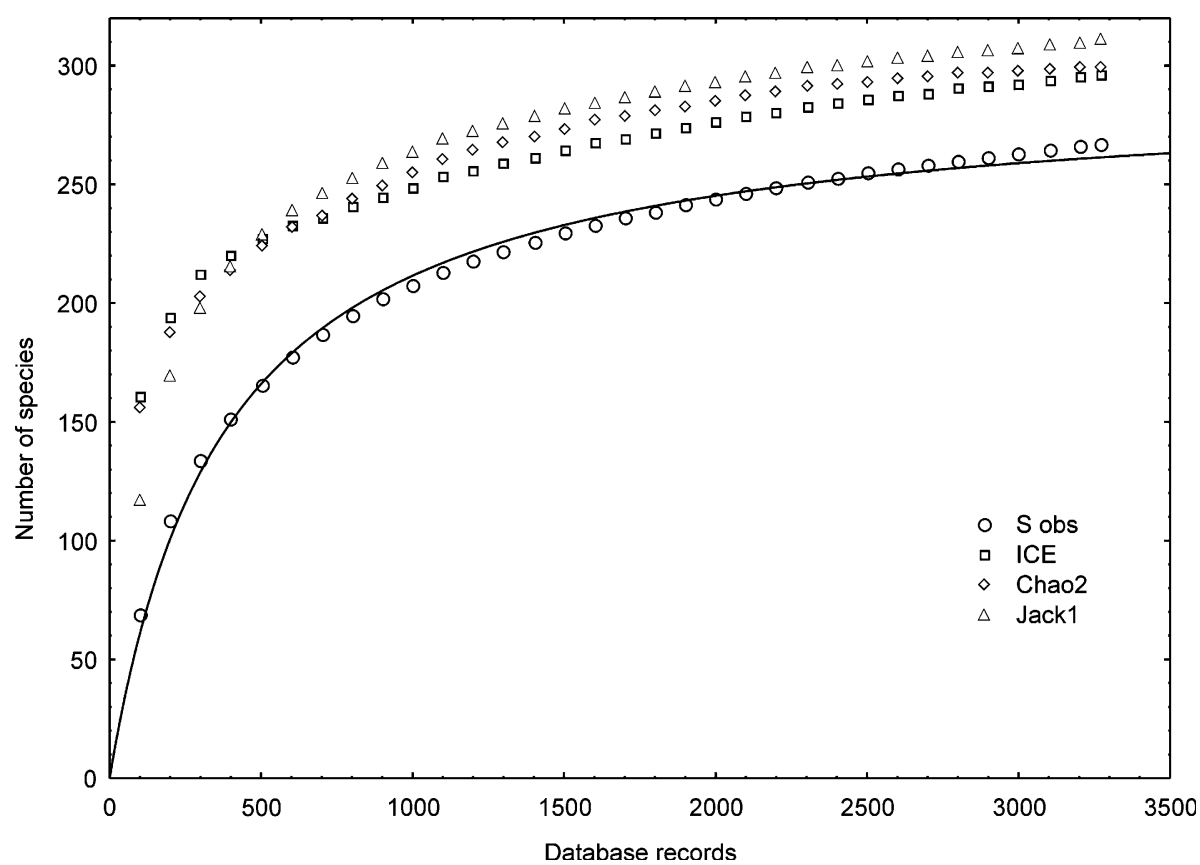

Figure 3. Species accumulation curve generated from the field study with the fitted Clench function and the non-parametric estimators ICE, Chao2 and first-order jackknife.

Table 1. Number of species recorded ( $S$ obs), estimates and percentage of the estimated value recorded for the long-term sampling, the whole historic inventory and the citations published before 1998.

\begin{tabular}{lccr}
\hline Method & S obs & Estimate & $\%$ \\
\hline Rarefaction curve & & & \\
Clench asymptote & 267 & 292 & 91.4 \\
ICE & 267 & 297 & 89.9 \\
Chao 2 & 267 & 300 & 89.0 \\
Jackknife 1 & 267 & 311 & 85.9 \\
Historic curves & & & 85.7 \\
Clench for 1991-2001 & 276 & 322 & 95.2 \\
Clench for 1996-2001 & 276 & 290 & 64.8 \\
Clench for citations published before 1998 & 83 & 128 & \\
\hline
\end{tabular}

in the rate of addition of new records due to the significant increase of sampling effort since 1991 to 2001. In this final section the historic curve becomes asymptotic. The fitted Clench function estimates an asymptote of 322 species for the curve between 1991 and 2001 (Figure 4b), and an asymptote of 290 species if the function is adjusted to the section between 1996 and 2001 

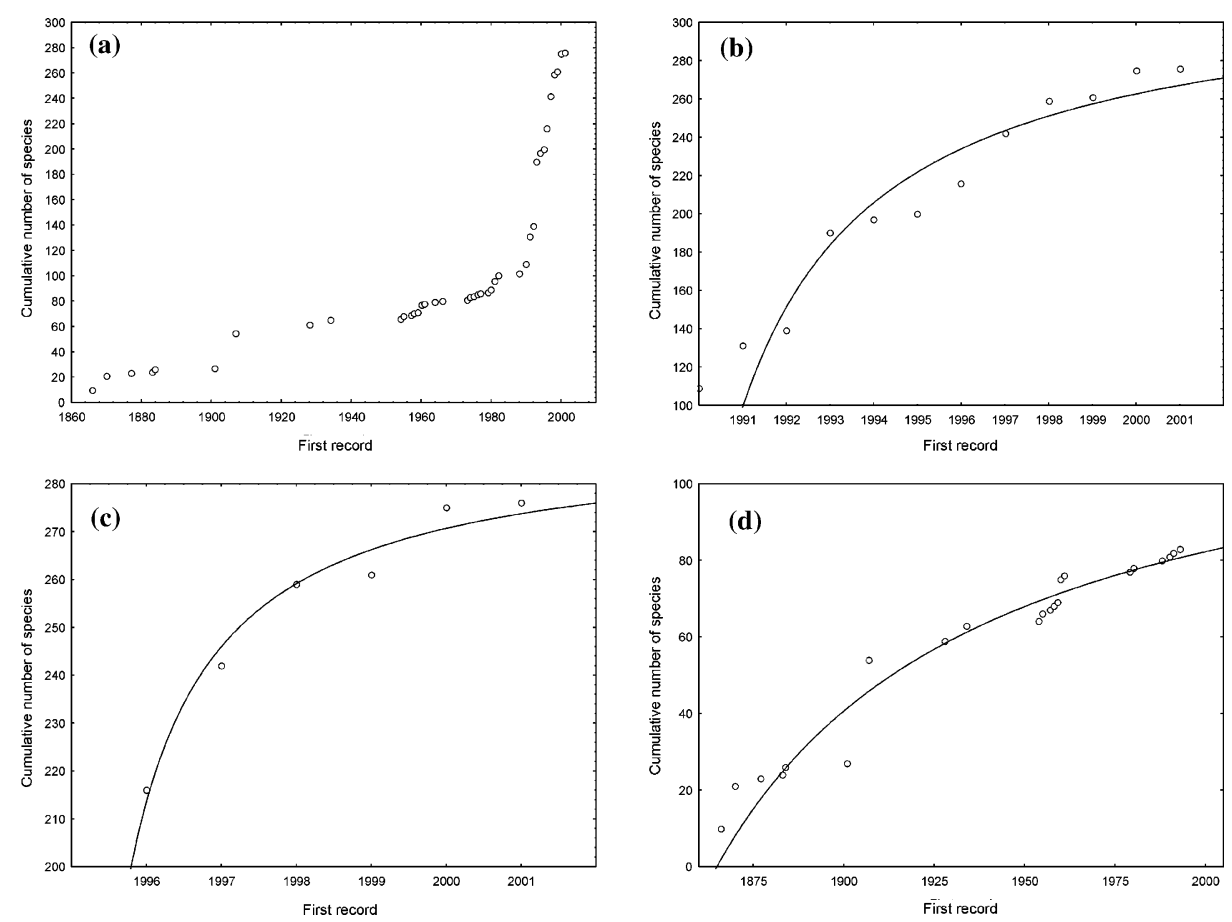

Figure 4. Cumulative number of species recorded per year. (a) Historic curve (1866-2001). (b) Fitted Clench function considering the final period with two collectors (1991-2001). (c) Fitted Clench function considering the final period with a single collector (1996-2001). (d) Cumulative number of citations published before 1998. The curve reaches an asymptote due to the lack of sampling effort and the unrealistic estimation is 128 species.

(Figure 4c). Since the recorded species richness is 276, the estimated completeness of the inventory reaches about $85.7-95.2 \%$ (Table 1).

\section{Discussion}

The current state of the inventory is considered reasonably complete, because the different estimation methods agree in their values. The Clench function and the non-parametric estimators computed from the sampling rarefaction curve, as well as the historic curve of the cumulative number of recorded species predict a percentage of undetected species between 5 and 15\% (14-44 species). The fauna of Chrysomelidae from Galicia $\left(29574 \mathrm{~km}^{2}\right)$ comprises 276 recorded species, but the estimated total richness reaches between 290 and 323. If these values are compared with those of other Iberian regions, Galician diversity is quite similar to the 339 species cited from Aragón $\left(47,6974 \mathrm{~km}^{2}\right)$, though this number might be an underestimation (Vives 2000), but it is far from the 384 taxa recorded from Cataluña $\left(32091 \mathrm{~km}^{2}\right)$ (Petitpierre 1994). The reduced species 
richness recorded in Galicia in comparison with Aragón or Cataluña may not reflect sampling deficiencies but real differences in leaf beetle diversity, since the species accumulation curves and the non-parametric estimators nearly reach the predicted values (85.9-95.1\% of the estimated richness). Both regions have a higher species richness than Galicia probably due to their marked environmental gradients between the high Pyrenees mountains and the Mediterranean or even arid plains and valleys, that allow the presence of both Eurosiberian and Mediterranean elements, as well as the presence of endemic Pyrenean species not distributed in other regions of the Iberian peninsula. In Galicia the Mediterranean phytogeographic region is restricted to a small southeastern area, and mountain ranges reach maximum altitudes up to $2000 \mathrm{~m}$, whereas in the Pyrenees there are more than 200 summits that rise above $3000 \mathrm{~m}$.

The description of two new species from Galicia and the citation of eight species new for the Iberian peninsula, indicate the previous poor knowledge about the faunas occurring in the northern regions of the Iberian peninsula and the need of further sampling effort in such areas (i.e. Asturias, Cantabria, País Vasco). This poor state of knowledge was particularly pronounced in Galicia, where records for the two thirds of the known species were published since 1998. In fact, the scarcity of published records before 1998 would have avoided even any reliable estimation of the number of species occurring in the region, because the cumulative curve (Figure 4d) reaches an asymptote due to the lack of sampling effort and not to the saturation of the inventory. Only 83 species were cited before 1998 and using these data the estimation generated by the Clench function is 128 species. This fact shows the importance of publishing faunistic inventories, which are the base for research on biodiversity and biogeography.

In summary, the study presented herein points out the need of extensive field work to collect highly diverse groups in still poorly surveyed territories, as well how this knowledge from non-standardized but extensive samplings can be used to get estimates of actual species richness. In the case of Galician leaf beetles, the long-term sampling has showed the previous poor knowledge of Iberian Eurosiberian-type faunas with the discovery of new taxa and the addition of many new records to the Galician and Iberian inventories. Also, the use of different methods in assessing the completeness of the inventory allows us to consider that estimates are accurate, because all non-parametric estimators, samplebased and historic curves predict values of total species richness within a narrow range. The recorded richness represent about $85-95 \%$ of the predicted values. Thus, the inventory seems to be reasonably complete and therefore valuable to describe biodiversity patterns of Iberian and European Chrysomelidae.

\section{Acknowledgements}

We are grateful to E. Petitpierre (Universitat de les Illes Balears, Palma de Mallorca) for valuable help on many taxonomic and bibliographic topics, G. Cerviño (A. Coruña) for the loan of his interesting collection of 
Chrysomelidae and S. Barallobre (Santiago de Compostela) for her collaboration in different expeditions to collect beetles. We also thank J.M. Lobo, J. Hortal and A. Jiménez-Valverde (Museo Nacional de Ciencias Naturales, Madrid) for helpful advice on statistical methods and valuable comments that greatly improved this paper. This study was supported by Xunta de Galicia with projects PGIDT01PXI20004PR and PGIDT01MAM20001PR, as well as $\mathrm{Ph} . \mathrm{D}$. and postdoctoral grants to A.B.

\section{Appendix A}

See Materials and methods section for explanation of terms.

\begin{tabular}{|c|c|c|c|c|}
\hline Species & $\begin{array}{l}\text { First } \\
\text { record }\end{array}$ & Published records & Main areas & Dist. \\
\hline $\begin{array}{l}\text { Donacia reticulata } \\
\text { (Gyllenhal) }\end{array}$ & 1866 & López Seoane (1866) & $\mathrm{Y}$ & WR \\
\hline $\begin{array}{l}\text { Donacia galaica } \\
\text { Báguena }\end{array}$ & 1959 & $\begin{array}{l}\text { Báguena (1959), } \\
\text { Petitpierre (2000) } \\
\text { and Baselga and } \\
\text { Novoa }(2002 b)\end{array}$ & $\mathrm{B}, \mathrm{H}, \mathrm{X}, \mathrm{Y}$ & Ibe \\
\hline $\begin{array}{l}\text { Donacia marginata } \\
\text { Hoppe }\end{array}$ & 1960 & $\begin{array}{l}\text { Báguena (1960a) and } \\
\text { Baselga and Novoa } \\
(1999 a, 2000 a)\end{array}$ & $\mathrm{A}, \mathrm{D}, \mathrm{G}, \mathrm{X}, \mathrm{Y}$ & WR \\
\hline $\begin{array}{l}\text { Donacia bicolora } \\
\text { Zschach }\end{array}$ & 1960 & Báguena (1960a) & $\mathrm{X}, \mathrm{Y}$ & WR \\
\hline $\begin{array}{l}\text { Donacia vulgaris } \\
\text { Zschach }\end{array}$ & 1992 & New record & $\mathrm{X}$ & WR \\
\hline $\begin{array}{l}\text { Donacia simplex } \\
\text { Fabricius }\end{array}$ & 1960 & Báguena (1960a) & $\mathrm{Y}$ & WR \\
\hline $\begin{array}{l}\text { Plateumaris sericea } \\
\text { (Linné) }\end{array}$ & 1960 & $\begin{array}{l}\text { Báguena (1960a) and } \\
\text { Baselga and Novoa } \\
(2000 a, b, 2002 b)\end{array}$ & $\mathrm{A}, \mathrm{B}, \mathrm{C}, \mathrm{D}, \mathrm{E}, \mathrm{F}, \mathrm{H}, \mathrm{X}, \mathrm{Y}$ & WR \\
\hline $\begin{array}{l}\text { Oulema erichsonii } \\
\text { (Suffrian) }\end{array}$ & 1990 & $\begin{array}{l}\text { Baselga and Novoa } \\
(1999 b, 2000 b, 2002 b)\end{array}$ & $\mathrm{E}, \mathrm{F}, \mathrm{G}, \mathrm{X}$ & Eur \\
\hline $\begin{array}{l}\text { Oulema gallaeciana } \\
\text { (Heyden) }\end{array}$ & 1870 & $\begin{array}{l}\text { Heyden (1870), Iglesias } \\
\text { (1928), Vives and } \\
\text { González (1994) and } \\
\text { Baselga and Novoa } \\
(1999 a, 2000 a, b, 2002 b)\end{array}$ & $\mathrm{A}, \mathrm{B}, \mathrm{C}, \mathrm{D}, \mathrm{E}, \mathrm{F}, \mathrm{H}, \mathrm{X}, \mathrm{Y}$ & Eur \\
\hline $\begin{array}{l}\text { Oulema hoffmannseggi } \\
\text { (Lacordaire) }\end{array}$ & 1993 & $\begin{array}{l}\text { Baselga and Novoa } \\
(2000 \mathrm{c})\end{array}$ & $\mathrm{X}$ & Med \\
\hline $\begin{array}{l}\text { Oulema melanopus } \\
\text { (Linné) }\end{array}$ & 1993 & $\begin{array}{l}\text { Baselga and Novoa } \\
(1999 \mathrm{a}, 2000 \mathrm{a}, \mathrm{b})\end{array}$ & $\mathrm{A}, \mathrm{B}, \mathrm{C}, \mathrm{D}, \mathrm{E}, \mathrm{F}, \mathrm{X}$ & WR \\
\hline $\begin{array}{l}\text { Oulema duftschmidi } \\
\text { (Redtenbacher) }\end{array}$ & 1993 & $\begin{array}{l}\text { Baselga and Novoa } \\
(2000 \mathrm{a}, \mathrm{b}, 2002 \mathrm{~b})\end{array}$ & $\mathrm{A}, \mathrm{B}, \mathrm{C}, \mathrm{D}, \mathrm{E}, \mathrm{F}, \mathrm{H}, \mathrm{X}$ & WR \\
\hline $\begin{array}{l}\text { Oulema rufocyanea } \\
\text { (Suffrian) }\end{array}$ & 2000 & $\begin{array}{l}\text { Baselga and Novoa } \\
(2002 \mathrm{~b})\end{array}$ & $\mathrm{H}$ & Eur \\
\hline
\end{tabular}


Appendix A Continued.

\begin{tabular}{|c|c|c|c|c|}
\hline Species & $\begin{array}{l}\text { First } \\
\text { record }\end{array}$ & Published records & Main areas & Dist. \\
\hline $\begin{array}{l}\text { Crioceris paracenthesis } \\
\text { (Linné) }\end{array}$ & 1997 & $\begin{array}{l}\text { Baselga and Novoa } \\
(2000 \mathrm{a})\end{array}$ & A & Med \\
\hline $\begin{array}{l}\text { Crioceris asparagi } \\
\text { (Linné) }\end{array}$ & 1928 & $\begin{array}{l}\text { Iglesias (1928), } \\
\text { González de Andrés } \\
\text { (1934) and Baselga and } \\
\text { Novoa (1999a, 2000a) }\end{array}$ & $\mathrm{A}, \mathrm{X}, \mathrm{Y}$ & WR \\
\hline $\begin{array}{l}\text { Lilioceris lilii (Scopoli) } \\
\text { ssp. laeviuscula (Weise) }\end{array}$ & 1907 & $\begin{array}{l}\text { Chapman and Champion } \\
\text { (1907) and Baselga and } \\
\text { Novoa (2002b) }\end{array}$ & $\mathrm{X}, \mathrm{Y}$ & WR \\
\hline $\begin{array}{l}\text { Labidostomis lusitanica } \\
\text { (Germar) }\end{array}$ & 1907 & $\begin{array}{l}\text { Chapman and Champion } \\
\text { (1907), Iglesias (1928), } \\
\text { Baselga and Novoa } \\
\text { (1999a, 2000b, 2002b) } \\
\text { and Petitpierre (2000) }\end{array}$ & $\mathrm{E}, \mathrm{F}, \mathrm{G}, \mathrm{X}, \mathrm{Y}$ & Med \\
\hline $\begin{array}{l}\text { Labidostomis taxicornis } \\
\text { (Fabricius) }\end{array}$ & 1974 & $\begin{array}{l}\text { Baselga and Novoa } \\
\text { (2001a) }\end{array}$ & $\mathrm{X}$ & Med \\
\hline $\begin{array}{l}\text { Lachnaia cylindrica } \\
\text { (Lacordaire) }\end{array}$ & 1998 & $\begin{array}{l}\text { Baselga and Novoa } \\
(2000 \mathrm{~b})\end{array}$ & $\mathrm{F}$ & Med \\
\hline $\begin{array}{l}\text { Lachnaia hirta } \\
\text { (Fabricius) }\end{array}$ & 1998 & $\begin{array}{l}\text { Baselga and Novoa } \\
(1999 a, 2000 b, 2002 b)\end{array}$ & $\mathrm{D}, \mathrm{F}, \mathrm{G}, \mathrm{X}$ & Med \\
\hline $\begin{array}{l}\text { Lachnaia pubescens } \\
\text { (Dufour) }\end{array}$ & 1907 & $\begin{array}{l}\text { Chapman and Champion } \\
\text { (1907), Iglesias (1928) } \\
\text { and Baselga and Novoa } \\
\text { (1999a, 2000b, 2002b) }\end{array}$ & E, F, G, H, X, Y & Med \\
\hline $\begin{array}{l}\text { Lachnaia tristigma } \\
\text { (Lacordaire) }\end{array}$ & 1907 & $\begin{array}{l}\text { Chapman and Champion } \\
\text { (1907) and Baselga and } \\
\text { Novoa (2002b) }\end{array}$ & $\mathrm{D}, \mathrm{E}, \mathrm{X}, \mathrm{Y}$ & Med \\
\hline $\begin{array}{l}\text { Tituboea biguttata } \\
\text { (Olivier) }\end{array}$ & 1994 & $\begin{array}{l}\text { Baselga and Novoa } \\
(2002 b)\end{array}$ & G & Med \\
\hline $\begin{array}{l}\text { Tituboea sexmaculata } \\
\text { (Fabricius) }\end{array}$ & 2000 & New record & G & Med \\
\hline $\begin{array}{l}\text { Clytra atraphaxidis } \\
\text { Pallas }\end{array}$ & 1907 & $\begin{array}{l}\text { Chapman and Champion } \\
\text { (1907) and Baselga and } \\
\text { Novoa (2002b) }\end{array}$ & Y & WR \\
\hline $\begin{array}{l}\text { Clytra quadripunctata } \\
\text { (Linné) ssp. } \\
\text { quadripunctata (Linné) }\end{array}$ & 1998 & $\begin{array}{l}\text { Baselga and Novoa } \\
(2000 \mathrm{~b})\end{array}$ & $\mathrm{F}$ & Eur \\
\hline $\begin{array}{l}\text { Clytra espanoli Daccordi } \\
\text { and Petitpierre }\end{array}$ & 1907 & $\begin{array}{l}\text { Chapman and Champion } \\
(1907) \text {, Iglesias (1928) } \\
\text { and Baselga and Novoa } \\
(1999 a, 2000 b, 2002 b)\end{array}$ & B, C, F, H, X, Y & Ibe \\
\hline $\begin{array}{l}\text { Smaragdina concolor } \\
\text { (Fabricius) }\end{array}$ & 1907 & $\begin{array}{l}\text { Chapman and Champion } \\
\text { (1907) and Baselga and } \\
\text { Novoa (1999a, 2000b, } \\
\text { 2002b) }\end{array}$ & $\mathrm{B}, \mathrm{C}, \mathrm{D}, \mathrm{E}, \mathrm{F}, \mathrm{G}, \mathrm{H}, \mathrm{X}, \mathrm{Y}$ & Med \\
\hline $\begin{array}{l}\text { Smaragdina reyi } \\
\text { (Brisout) }\end{array}$ & 1960 & $\begin{array}{l}\text { Báguena (1960b), Cobos } \\
\text { (1969), Baselga and } \\
\text { Novoa (2000b, 2002b) } \\
\text { and Petitpierre (2000) }\end{array}$ & $\mathrm{B}, \mathrm{C}, \mathrm{D}, \mathrm{E}, \mathrm{F}, \mathrm{G}, \mathrm{H}, \mathrm{X}, \mathrm{Y}$ & Ibe \\
\hline
\end{tabular}


Appendix A Continued.

\begin{tabular}{|c|c|c|c|c|}
\hline Species & $\begin{array}{l}\text { First } \\
\text { record }\end{array}$ & Published records & Main areas & Dist. \\
\hline $\begin{array}{l}\text { Coptocephala brevicornis } \\
\text { (Lefévre) }\end{array}$ & 1960 & $\begin{array}{l}\text { Báguena (1960c) and } \\
\text { Baselga and Novoa } \\
(1999 a, 2000 a, 2002 b)\end{array}$ & $\mathrm{A}, \mathrm{G}, \mathrm{X}, \mathrm{Y}$ & Ibe \\
\hline $\begin{array}{l}\text { Coptocephala scopolina } \\
\text { (Linné) }\end{array}$ & 1981 & $\begin{array}{l}\text { Baselga and Novoa } \\
(2000 \mathrm{a}, 2002 \mathrm{~b})\end{array}$ & $\mathrm{A}, \mathrm{G}, \mathrm{H}, \mathrm{X}$ & Med \\
\hline $\begin{array}{l}\text { Stylosomus ilicicola } \\
\text { Suffrian }\end{array}$ & 1961 & Codina Padilla (1961a) & $\mathrm{Y}$ & Med \\
\hline $\begin{array}{l}\text { Stylosomus rugithorax } \\
\text { Abeille }\end{array}$ & 1907 & $\begin{array}{l}\text { Baselga and Novoa } \\
(2000 \mathrm{~b}, 2002 \mathrm{~b})\end{array}$ & $\mathrm{B}, \mathrm{C}, \mathrm{D}, \mathrm{E}, \mathrm{F}, \mathrm{H}, \mathrm{X}$ & Ibe \\
\hline $\begin{array}{l}\text { Pachybrachis pteromelas } \\
\text { Graëlls }\end{array}$ & 2000 & New record & G & Ibe \\
\hline $\begin{array}{l}\text { Pachybrachis hippophaes } \\
\text { Suffrian }\end{array}$ & 1907 & $\begin{array}{l}\text { Chapman and Champion } \\
\text { (1907) and Baselga and } \\
\text { Novoa (2002b) }\end{array}$ & Y & Eur \\
\hline $\begin{array}{l}\text { Pachybrachis azureus } \\
\text { Suffrian }\end{array}$ & 1907 & $\begin{array}{l}\text { Chapman and Champion } \\
\text { (1907) and Baselga and } \\
\text { Novoa (1999b, 2000b, } \\
\text { 2002b) }\end{array}$ & $\mathrm{D}, \mathrm{F}, \mathrm{G}, \mathrm{H}, \mathrm{X}, \mathrm{Y}$ & Med \\
\hline $\begin{array}{l}\text { Cryptocephalus excisus } \\
\text { Seidlitz }\end{array}$ & 1998 & $\begin{array}{l}\text { Baselga and Novoa } \\
(2000 \mathrm{~b})\end{array}$ & $\mathrm{F}$ & Ibe \\
\hline $\begin{array}{l}\text { Cryptocephalus lusitanicus } \\
\text { Suffrian }\end{array}$ & 1907 & $\begin{array}{l}\text { Chapman and Champion } \\
\text { (1907) and Baselga and } \\
\text { Novoa (2000b, 2002b) }\end{array}$ & E, F, G, X, Y & Ibe \\
\hline $\begin{array}{l}\text { Cryptocephalus pominorum } \\
\text { Burlini }\end{array}$ & 1907 & $\begin{array}{l}\text { Chapman and Champion } \\
\text { (1907), Bourdonné (1994) } \\
\text { and Baselga and } \\
\text { Novoa }(2000 \mathrm{~b}, 2002 \mathrm{~b})\end{array}$ & $\mathrm{F}, \mathrm{G}, \mathrm{X}, \mathrm{Y}$ & Ibe \\
\hline $\begin{array}{l}\text { Cryptocephalus pexicollis } \\
\text { Suffrian }\end{array}$ & 1955 & Burlini (1955) & $\mathrm{Y}$ & Med \\
\hline $\begin{array}{l}\text { Cryptocephalus obliteratifer } \\
\text { Pic }\end{array}$ & 1907 & $\begin{array}{l}\text { Chapman and Champion } \\
\text { (1907) and Baselga and } \\
\text { Novoa (2002b) }\end{array}$ & G, Y & Med \\
\hline $\begin{array}{l}\text { Cryptocephalus bipunctatus } \\
\text { (Linné) }\end{array}$ & 1980 & $\begin{array}{l}\text { Chapman and Champion } \\
\text { (1907) and Baselga and } \\
\text { Novoa (2000b, 2002b) }\end{array}$ & C, F, G, X, Y & Eur \\
\hline $\begin{array}{l}\text { Cryptocephalus rugicollis } \\
\text { Olivier }\end{array}$ & 2000 & $\begin{array}{l}\text { Baselga and Novoa } \\
(2002 b)\end{array}$ & G & Med \\
\hline $\begin{array}{l}\text { Cryptocephalus aureolus } \\
\text { Suffrian }\end{array}$ & 1982 & $\begin{array}{l}\text { Baselga and Novoa } \\
\text { (1999a, 2000a, b, d, } \\
\text { 2002b) }\end{array}$ & $\mathrm{A}, \mathrm{B}, \mathrm{C}, \mathrm{D}, \mathrm{F}, \mathrm{X}$ & Eur \\
\hline $\begin{array}{l}\text { Cryptocephalus globicollis } \\
\text { Suffrian }\end{array}$ & 1907 & $\begin{array}{l}\text { Chapman and Champion } \\
\text { (1907) and Baselga and } \\
\text { Novoa (2002b) }\end{array}$ & $\mathrm{X}, \mathrm{Y}$ & Med \\
\hline $\begin{array}{l}\text { Cryptocephalus cantabricus } \\
\text { Franz }\end{array}$ & 1958 & $\begin{array}{l}\text { Franz (1958) and } \\
\text { Baselga and Novoa } \\
(2000 \mathrm{~b}, \mathrm{~d}, 2002 \mathrm{~b})\end{array}$ & $\mathrm{B}, \mathrm{C}, \mathrm{E}, \mathrm{F}, \mathrm{G}, \mathrm{H}, \mathrm{X}, \mathrm{Y}$ & Ibe \\
\hline $\begin{array}{l}\text { Cryptocephalus violaceus } \\
\text { Laicharting }\end{array}$ & 1907 & $\begin{array}{l}\text { Chapman and Champion } \\
(1907) \text {, Petitpierre (2000) } \\
\text { and Baselga and Novoa } \\
(2002 b)\end{array}$ & Y & Eur \\
\hline
\end{tabular}


Appendix A Continued.

\begin{tabular}{|c|c|c|c|c|}
\hline Species & $\begin{array}{l}\text { First } \\
\text { record }\end{array}$ & Published records & Main areas & Dist. \\
\hline $\begin{array}{l}\text { Cryptocephalus tibialis } \\
\text { Brisout }\end{array}$ & 1955 & $\begin{array}{l}\text { Burlini (1955) and } \\
\text { Baselga and Novoa } \\
(1999 a, 2000 b, 2002 b)\end{array}$ & $\mathrm{E}, \mathrm{F}, \mathrm{G}, \mathrm{H}, \mathrm{X}, \mathrm{Y}$ & Eur \\
\hline $\begin{array}{l}\text { Cryptocephalus parvulus } \\
\text { Müller }\end{array}$ & 2000 & New record & $\mathrm{X}$ & WR \\
\hline $\begin{array}{l}\text { Cryptocephalus } \\
\text { androgyne Marseul ssp. } \\
\text { pelleti Marseul }\end{array}$ & 1993 & New record & $\mathrm{C}, \mathrm{X}$ & Eur \\
\hline $\begin{array}{l}\text { Cryptocephalus cynarae } \\
\text { Suffrian }\end{array}$ & 1866 & $\begin{array}{l}\text { López Seoane (1866), } \\
\text { Chapman and Champion } \\
\text { (1907) and Baselga and } \\
\text { Novoa (1999a, 2002b) }\end{array}$ & $\mathrm{D}, \mathrm{G}, \mathrm{X}, \mathrm{Y}$ & Ibe \\
\hline $\begin{array}{l}\text { Cryptocephalus moraei } \\
\text { (Linné) }\end{array}$ & 1907 & $\begin{array}{l}\text { Chapman and Champion } \\
\text { (1907) and Baselga and } \\
\text { Novoa (2002b) }\end{array}$ & $\mathrm{B}, \mathrm{D}, \mathrm{X}, \mathrm{Y}$ & Eur \\
\hline $\begin{array}{l}\text { Cryptocephalus crassus } \\
\text { Olivier }\end{array}$ & 1907 & $\begin{array}{l}\text { Chapman and Champion } \\
\text { (1907) and Baselga and } \\
\text { Novoa (2002b) }\end{array}$ & $\mathrm{G}, \mathrm{Y}$ & Med \\
\hline $\begin{array}{l}\text { Cryptocephalus octoguttatus } \\
\text { (Linné) }\end{array}$ & 1992 & $\begin{array}{l}\text { Baselga and Novoa } \\
(1999 a, 2000 b, 2002 b)\end{array}$ & $\mathrm{C}, \mathrm{D}, \mathrm{E}, \mathrm{F}, \mathrm{G}, \mathrm{H}, \mathrm{X}$ & Med \\
\hline $\begin{array}{l}\text { Cryptocephalus vittatus } \\
\text { Fabricius }\end{array}$ & 1870 & $\begin{array}{l}\text { Heyden (1870), } \\
\text { Chapman and Champion } \\
\text { (1907), Iglesias (1928), } \\
\text { Burlini (1955) and } \\
\text { Baselga and Novoa } \\
\text { (1999a, 2000a, b, 2002b) }\end{array}$ & $\mathrm{A}, \mathrm{B}, \mathrm{C}, \mathrm{D}, \mathrm{E}, \mathrm{F}, \mathrm{G}, \mathrm{X}, \mathrm{Y}$ & WR \\
\hline Cryptocephalus celtibericus & 1877 & López Seoane (1877) and & $F, G, Y$ & Med \\
\hline Suffrian & & $\begin{array}{l}\text { Baselga and Novoa } \\
(2000 \mathrm{~b}, 2002 \mathrm{~b})\end{array}$ & & \\
\hline $\begin{array}{l}\text { Cryptocephalus bilineatus } \\
\text { (Linné) }\end{array}$ & 1870 & $\begin{array}{l}\text { Heyden (1870), } \\
\text { Plaza Infante (1979) } \\
\text { and Baselga and Novoa } \\
(2000 \mathrm{~b}, 2002 \mathrm{~b})\end{array}$ & $\mathrm{C}, \mathrm{F}, \mathrm{H}, \mathrm{X}, \mathrm{Y}$ & WR \\
\hline $\begin{array}{l}\text { Cryptocephalus mystacatus } \\
\text { Suffrian }\end{array}$ & 1907 & $\begin{array}{l}\text { Chapman and Champion } \\
\text { (1907) and Baselga and } \\
\text { Novoa (2000b, 2002b) }\end{array}$ & $\mathrm{D}, \mathrm{E}, \mathrm{F}, \mathrm{G}, \mathrm{H}, \mathrm{X}, \mathrm{Y}$ & Ibe \\
\hline $\begin{array}{l}\text { Cryptocephalus labiatus } \\
\text { (Linné) }\end{array}$ & 1991 & $\begin{array}{l}\text { Baselga and Novoa } \\
(1999 b, 2000 b)\end{array}$ & $\mathrm{C}, \mathrm{F}, \mathrm{X}$ & Eur \\
\hline $\begin{array}{l}\text { Cryptocephalus pygmaeus } \\
\text { Fabricius }\end{array}$ & 1907 & $\begin{array}{l}\text { Chapman and Champion } \\
\text { (1907) and Baselga and } \\
\text { Novoa (2000a, b, 2002b) }\end{array}$ & A, G, X, Y & WR \\
\hline $\begin{array}{l}\text { Cryptocephalus fulvus } \\
\text { (Goeze) }\end{array}$ & 1994 & $\begin{array}{l}\text { Baselga and Novoa } \\
(2000 \mathrm{a}, 2002 \mathrm{~b})\end{array}$ & $\mathrm{A}, \mathrm{G}, \mathrm{H}, \mathrm{X}$ & WR \\
\hline $\begin{array}{l}\text { Cryptocephalus macellus } \\
\text { Suffrian }\end{array}$ & 1979 & Plaza Infante (1979) & Y & Eur \\
\hline $\begin{array}{l}\text { Cryptocephalus pusillus } \\
\text { Fabricius }\end{array}$ & 1991 & New record & $\mathrm{C}, \mathrm{X}$ & Eur \\
\hline $\begin{array}{l}\text { Cryptocephalus rufipes } \\
\text { (Goeze) }\end{array}$ & 1991 & $\begin{array}{l}\text { Baselga and Novoa } \\
(2000 \mathrm{a}, \mathrm{b}, 2002 \mathrm{~b})\end{array}$ & $\mathrm{A}, \mathrm{B}, \mathrm{C}, \mathrm{D}, \mathrm{E}, \mathrm{F}, \mathrm{H}, \mathrm{X}$ & WR \\
\hline
\end{tabular}


Appendix A Continued.

\begin{tabular}{|c|c|c|c|c|}
\hline Species & $\begin{array}{l}\text { First } \\
\text { record }\end{array}$ & Published records & Main areas & Dist. \\
\hline $\begin{array}{l}\text { Oomorphus concolor } \\
\text { (Sturm) }\end{array}$ & 1998 & $\begin{array}{l}\text { Baselga and Novoa } \\
(1999 b, 2000 b)\end{array}$ & $\mathrm{C}, \mathrm{F}$ & Eur \\
\hline $\begin{array}{l}\text { Bromius obscurus } \\
\text { (Linné) }\end{array}$ & 2000 & $\begin{array}{l}\text { Baselga and Novoa } \\
\text { (2001a, 2002b) }\end{array}$ & $\mathrm{H}$ & WR \\
\hline $\begin{array}{l}\text { Timarcha calceata } \\
\text { Pérez Arcas }\end{array}$ & 1975 & $\begin{array}{l}\text { Baselga and Novoa } \\
(1999 a, 2000 b)\end{array}$ & $\mathrm{F}, \mathrm{X}$ & Ibe \\
\hline $\begin{array}{l}\text { Timarcha geniculata } \\
\text { (Germar) }\end{array}$ & 1977 & $\begin{array}{l}\text { Baselga and Novoa } \\
(2002 \mathrm{~b})\end{array}$ & $\mathrm{F}, \mathrm{H}, \mathrm{Y}$ & Ibe \\
\hline $\begin{array}{l}\text { Timarcha asturiensis } \\
\text { Kraatz }\end{array}$ & 1883 & $\begin{array}{l}\text { Marseul (1883), Bechyné } \\
\text { (1948) and Baselga and } \\
\text { Novoa (2000a) }\end{array}$ & A, Y & Ibe \\
\hline $\begin{array}{l}\text { Timarcha chloropus } \\
\text { (Germar) }\end{array}$ & 1884 & $\begin{array}{l}\text { Fairmaire (1884), } \\
\text { Bechyné (1948), Vives } \\
\text { and González (1998) } \\
\text { and Baselga and Novoa } \\
(1999 a, 2000 a)\end{array}$ & $\mathrm{A}, \mathrm{D}, \mathrm{X}, \mathrm{Y}$ & Ibe \\
\hline $\begin{array}{l}\text { Timarcha gougeleti } \\
\text { Fairmaire }\end{array}$ & 1877 & $\begin{array}{l}\text { López Seoane (1877), } \\
\text { Fairmaire (1884), } \\
\text { Bechyné (1948), Vives } \\
\text { and González (1998) and } \\
\text { Baselga and Novoa } \\
(1999 a, 2000 a)\end{array}$ & $\mathrm{A}, \mathrm{B}, \mathrm{C}, \mathrm{X}, \mathrm{Y}$ & Ibe \\
\hline $\begin{array}{l}\text { Timarcha trapezicollis } \\
\text { Fairmaire }\end{array}$ & 1884 & Fairmaire (1884) & $\mathrm{Y}$ & Ibe \\
\hline $\begin{array}{l}\text { Leptinotarsa decemlineata } \\
\text { Say }\end{array}$ & 1993 & $\begin{array}{l}\text { Baselga and Novoa } \\
(1999 a, 2000 a, 2002 b)\end{array}$ & A, B, H, X & Intr. \\
\hline $\begin{array}{l}\text { Chrysolina herbacea } \\
\text { (Duftschmid) }\end{array}$ & 1866 & $\begin{array}{l}\text { Baselga and Novoa } \\
(1999 a, 2000 a, b, 2002 b)\end{array}$ & $\mathrm{A}, \mathrm{B}, \mathrm{D}, \mathrm{E}, \mathrm{F}, \mathrm{G}, \mathrm{H}, \mathrm{X}$ & WR \\
\hline $\begin{array}{l}\text { Chrysolina fastuosa } \\
\text { (Scopoli) }\end{array}$ & 1996 & $\begin{array}{l}\text { Baselga and Novoa } \\
(1999 \mathrm{a}, 2002 \mathrm{~b})\end{array}$ & $\mathrm{B}, \mathrm{H}, \mathrm{X}$ & WR \\
\hline $\begin{array}{l}\text { Chrysolina polita } \\
\text { (Linné) }\end{array}$ & 1982 & $\begin{array}{l}\text { Baselga and Novoa } \\
(1999 \mathrm{a}, 2000 \mathrm{a}, \mathrm{b})\end{array}$ & $\mathrm{A}, \mathrm{D}, \mathrm{F}, \mathrm{X}$ & Eur \\
\hline $\begin{array}{l}\text { Chrysolina bankii } \\
\text { (Fabricius) }\end{array}$ & 1928 & $\begin{array}{l}\text { Iglesias }(1928) \text { and } \\
\text { Baselga and Novoa } \\
(1999 \mathrm{a}, 2000 \mathrm{a}, \mathrm{b})\end{array}$ & $\mathrm{A}, \mathrm{B}, \mathrm{C}, \mathrm{D}, \mathrm{F}, \mathrm{X}, \mathrm{Y}$ & Med \\
\hline $\begin{array}{l}\text { Chrysolina americana } \\
\text { (Linné) }\end{array}$ & 1907 & $\begin{array}{l}\text { Chapman and Champion } \\
(1907) \text {, Iglesias (1928) } \\
\text { and Baselga and Novoa } \\
(1999 a, 2002 b)\end{array}$ & G, X, Y & Med \\
\hline $\begin{array}{l}\text { Chrysolina rufoaenea } \\
\text { (Suffrian) }\end{array}$ & 1991 & New record & $\mathrm{D}, \mathrm{E}, \mathrm{X}$ & Eur \\
\hline $\begin{array}{l}\text { Chrysolina haemoptera } \\
\text { (Linné) }\end{array}$ & 1866 & $\begin{array}{l}\text { López Seoane (1866), } \\
\text { Iglesias (1928) and } \\
\text { Baselga and Novoa } \\
\text { (1999a, 2000a, b, 2002b) }\end{array}$ & $\mathrm{A}, \mathrm{C}, \mathrm{D}, \mathrm{H}, \mathrm{X}, \mathrm{Y}$ & Eur \\
\hline $\begin{array}{l}\text { Chrysolina mactata } \\
\text { (Fairmaire) }\end{array}$ & 1866 & $\begin{array}{l}\text { López Seoane (1866), } \\
\text { López Seoane (1877) and } \\
\text { Baselga and Novoa } \\
(1998,1999 a, 2000 b)\end{array}$ & C, F, X, Y & Ibe \\
\hline
\end{tabular}


Appendix A Continued.

\begin{tabular}{|c|c|c|c|c|}
\hline Species & $\begin{array}{l}\text { First } \\
\text { record }\end{array}$ & Published records & Main areas & Dist. \\
\hline $\begin{array}{l}\text { Chrysolina gypsophilae } \\
\text { (Küster) }\end{array}$ & 1928 & $\begin{array}{l}\text { Baselga and Novoa } \\
\text { (1999a, 2000a) }\end{array}$ & $A, G$ & WR \\
\hline $\begin{array}{l}\text { Chrysolina latecincta } \\
\text { (Demaison) ssp. } \\
\text { decipiens (Franz) }\end{array}$ & 1988 & $\begin{array}{l}\text { Petitpierre (1988) and } \\
\text { Baselga and Novoa } \\
(2002 \mathrm{~b})\end{array}$ & $\mathrm{X}, \mathrm{Y}$ & Eur \\
\hline $\begin{array}{l}\text { Chrysolina affinis } \\
\text { (Fabricius) ssp. } \\
\text { rufofemorata (Heyden) }\end{array}$ & 1973 & New record & $\mathrm{X}$ & Med \\
\hline $\begin{array}{l}\text { Chrysolina grossa } \\
\text { (Fabricius) ssp. } \\
\text { chloromaura (Olivier) }\end{array}$ & 1866 & $\begin{array}{l}\text { López Seoane (1866), } \\
\text { Iglesias (1928), Vives and } \\
\text { González (1998) and } \\
\text { Baselga and Novoa } \\
(1999 a, 2000 a)\end{array}$ & A, D, X, Y & Med \\
\hline $\begin{array}{l}\text { Chrysolina lucida } \\
\text { (Olivier) ssp. } \\
\text { torresi Bechyné }\end{array}$ & 1991 & $\begin{array}{l}\text { Baselga and Novoa } \\
(1999 a, 2000 a)\end{array}$ & $\mathrm{A}, \mathrm{X}$ & Eur \\
\hline $\begin{array}{l}\text { Chrysolina diluta } \\
\text { (Germar) }\end{array}$ & 1907 & $\begin{array}{l}\text { Chapman and Champion } \\
\text { (1907), Codina Padilla } \\
\text { (1961b) and Baselga and } \\
\text { Novoa (1999a, 2000a, } \\
\text { 2002b) }\end{array}$ & A, X, Y & Ibe \\
\hline $\begin{array}{l}\text { Chrysolina varians } \\
\text { (Schaller) }\end{array}$ & 1907 & $\begin{array}{l}\text { Baselga and Novoa } \\
(2000 \mathrm{~b})\end{array}$ & $\mathrm{F}$ & Eur \\
\hline $\begin{array}{l}\text { Chrysolina brunsvicensis } \\
\text { (Gravenhorst) }\end{array}$ & 1996 & $\begin{array}{l}\text { Baselga and Novoa } \\
(1999 b, 2000 b)\end{array}$ & $\mathrm{B}, \mathrm{D}, \mathrm{F}, \mathrm{X}$ & Eur \\
\hline $\begin{array}{l}\text { Chrysolina quadrigemina } \\
\text { (Suffrian) }\end{array}$ & 1991 & $\begin{array}{l}\text { Baselga and Novoa } \\
(2000 \mathrm{a}, \mathrm{b}, 2002 \mathrm{~b})\end{array}$ & $\mathrm{A}, \mathrm{B}, \mathrm{F}, \mathrm{G}, \mathrm{X}$ & WR \\
\hline $\begin{array}{l}\text { Chrysolina interstincta } \\
\text { (Suffrian) ssp. } \\
\text { graellsi (Pérez Arcas) }\end{array}$ & 1992 & $\begin{array}{l}\text { Baselga and Novoa } \\
(2000 \mathrm{c}, 2002 \mathrm{~b})\end{array}$ & $\mathrm{B}, \mathrm{E}, \mathrm{H}, \mathrm{X}$ & Med \\
\hline $\begin{array}{l}\text { Oreina alpestris } \\
\text { (Schummel) }\end{array}$ & 1981 & $\begin{array}{l}\text { Baselga and Novoa } \\
(2000 \mathrm{~b}, \mathrm{c})\end{array}$ & $\mathrm{F}, \mathrm{X}$ & Eur \\
\hline $\begin{array}{l}\text { Oreina ganglbaueri } \\
\text { (Jakob) }\end{array}$ & 1998 & New record & $\mathrm{F}$ & Ibe \\
\hline $\begin{array}{l}\text { Gastrophysa polygoni } \\
\text { (Linné) }\end{array}$ & 1990 & $\begin{array}{l}\text { Baselga and Novoa } \\
(2000 \mathrm{a})\end{array}$ & $\mathrm{A}, \mathrm{B}, \mathrm{G}, \mathrm{X}$ & WR \\
\hline $\begin{array}{l}\text { Gastrophysa unicolor } \\
\text { (Marsham) }\end{array}$ & 1866 & $\begin{array}{l}\text { López Seoane (1866) and } \\
\text { Baselga and Novoa } \\
(1999 a, 2000 a, b, 2002 b)\end{array}$ & $\mathrm{A}, \mathrm{B}, \mathrm{C}, \mathrm{D}, \mathrm{E}, \mathrm{F}, \mathrm{G}, \mathrm{H}, \mathrm{X}, \mathrm{Y}$ & Ibe \\
\hline $\begin{array}{l}\text { Colaspidema atrum } \\
\text { (Olivier) }\end{array}$ & 1934 & $\begin{array}{l}\text { González de Andrés } \\
\text { (1934) }\end{array}$ & $\mathrm{Y}$ & WR \\
\hline $\begin{array}{l}\text { Colaspidema dufouri } \\
\text { (P'érez Arcas) }\end{array}$ & 1993 & $\begin{array}{l}\text { Baselga and Novoa } \\
(2000 \mathrm{c})\end{array}$ & $\mathrm{X}$ & Ibe \\
\hline $\begin{array}{l}\text { Phaedon tumidulus } \\
\text { (Germar) }\end{array}$ & 1901 & $\begin{array}{l}\text { Bedel (1901) and Baselga } \\
\text { and Novoa }(2000 a, b)\end{array}$ & $\mathrm{A}, \mathrm{F}$ & WR \\
\hline $\begin{array}{l}\text { Phaedon cochleariae } \\
\text { (Fabricius) }\end{array}$ & 1999 & $\begin{array}{l}\text { Baselga and Novoa } \\
(2000 \mathrm{a}, 2002 \mathrm{~b})\end{array}$ & $\mathrm{A}, \mathrm{H}$ & WR \\
\hline $\begin{array}{l}\text { Phaedon armoraciae } \\
\text { (Linné) }\end{array}$ & 1998 & New record & E, $X$ & WR \\
\hline
\end{tabular}


Appendix A Continued.

\begin{tabular}{|c|c|c|c|c|}
\hline Species & $\begin{array}{l}\text { First } \\
\text { record }\end{array}$ & Published records & Main areas & Dist. \\
\hline $\begin{array}{l}\text { Cyrtonus corruscans } \\
\text { Vuillefroy }\end{array}$ & 1994 & New record & $\mathrm{X}$ & Ibe \\
\hline $\begin{array}{l}\text { Cyrtonus cupreovirens } \\
\text { Pérez Arcas }\end{array}$ & 1998 & New record & $\mathrm{D}$ & Ibe \\
\hline $\begin{array}{l}\text { Cyrtonus franzi } \\
\text { Cobos }\end{array}$ & 1954 & Cobos 1954 & $\mathrm{Y}$ & Ibe \\
\hline $\begin{array}{l}\text { Hydrothassa glabra } \\
\text { (Herbst) }\end{array}$ & 1993 & $\begin{array}{l}\text { Baselga and Novoa } \\
(2000 \mathrm{a}, \mathrm{b}, 2002 \mathrm{~b})\end{array}$ & $\mathrm{A}, \mathrm{B}, \mathrm{D}, \mathrm{F}, \mathrm{H}, \mathrm{X}$ & Eur \\
\hline $\begin{array}{l}\text { Hydrothassa fairmairei } \\
\text { (Brisout) }\end{array}$ & 1996 & $\begin{array}{l}\text { Baselga and Novoa } \\
(2000 \mathrm{a}, \mathrm{b})\end{array}$ & $\mathrm{A}, \mathrm{B}, \mathrm{C}, \mathrm{D}, \mathrm{F}, \mathrm{X}$ & Ibe \\
\hline $\begin{array}{l}\text { Prasocuris junci } \\
\text { (Brahm) }\end{array}$ & 1982 & $\begin{array}{l}\text { Baselga and Novoa } \\
(2000 \mathrm{a}, \mathrm{b})\end{array}$ & $\mathrm{A}, \mathrm{B}, \mathrm{F}, \mathrm{G}, \mathrm{X}$ & WR \\
\hline $\begin{array}{l}\text { Prasocuris phellandrii } \\
\text { (Linné) }\end{array}$ & 2001 & New record & 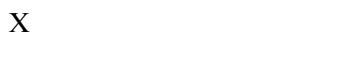 & WR \\
\hline $\begin{array}{l}\text { Plagiodera versicolora } \\
\text { (Laicharting) }\end{array}$ & 1907 & $\begin{array}{l}\text { Chapman and Champion } \\
\text { (1907), González de } \\
\text { Andrés (1934), Vives and } \\
\text { González (1998) and } \\
\text { Baselga and Novoa } \\
\text { (2000b, 2002b) }\end{array}$ & $\mathrm{B}, \mathrm{C}, \mathrm{D}, \mathrm{E}, \mathrm{F}, \mathrm{H}, \mathrm{X}, \mathrm{Y}$ & WR \\
\hline $\begin{array}{l}\text { Plagiosterna aenea } \\
\text { (Linné) }\end{array}$ & 1991 & $\begin{array}{l}\text { Baselga and Novoa } \\
(1999 \mathrm{a}, 2000 \mathrm{~b})\end{array}$ & $\mathrm{B}, \mathrm{F}, \mathrm{G}, \mathrm{X}$ & WR \\
\hline $\begin{array}{l}\text { Chrysomela populi } \\
\text { Linné }\end{array}$ & 1928 & $\begin{array}{l}\text { Iglesias (1928), González } \\
\text { de Andrés (1934) and } \\
\text { Baselga and Novoa } \\
(1999 a, 2000 b)\end{array}$ & $\mathrm{B}, \mathrm{F}, \mathrm{X}, \mathrm{Y}$ & WR \\
\hline $\begin{array}{l}\text { Gonioctena aegrota } \\
\text { Fabricius }\end{array}$ & 2000 & New record & G & Med \\
\hline $\begin{array}{l}\text { Gonioctena leprieuri } \\
(\mathrm{Pic})\end{array}$ & 1957 & $\begin{array}{l}\text { Bechyné (1957) and } \\
\text { Baselga and Novoa } \\
(2002 b)\end{array}$ & $\mathrm{F}, \mathrm{G}, \mathrm{H}$ & Ibe \\
\hline $\begin{array}{l}\text { Gonioctena olivacea } \\
\text { (Forster) }\end{array}$ & 1991 & $\begin{array}{l}\text { Baselga and Novoa } \\
(1999 a, 2000 a, b, 2002 b)\end{array}$ & $\mathrm{A}, \mathrm{B}, \mathrm{C}, \mathrm{D}, \mathrm{E}, \mathrm{F}, \mathrm{G}, \mathrm{H}, \mathrm{X}$ & WR \\
\hline $\begin{array}{l}\text { Phratora vulgatissima } \\
\text { (Linné) }\end{array}$ & 1991 & $\begin{array}{l}\text { Baselga and Novoa } \\
(1999 b)\end{array}$ & $\mathrm{B}, \mathrm{C}, \mathrm{D}, \mathrm{X}$ & WR \\
\hline $\begin{array}{l}\text { Phratora tibialis } \\
\text { (Suffrian) }\end{array}$ & 1988 & $\begin{array}{l}\text { Vives and González } \\
\text { (1988) }\end{array}$ & $\mathrm{Y}$ & Eur \\
\hline $\begin{array}{l}\text { Phratora laticollis } \\
\text { (Suffrian) }\end{array}$ & 1998 & $\begin{array}{l}\text { Baselga and Novoa } \\
(2000 \mathrm{~b})\end{array}$ & $\mathrm{F}$ & WR \\
\hline $\begin{array}{l}\text { Phratora vitellinae } \\
\text { (Linné) }\end{array}$ & 1998 & $\begin{array}{l}\text { Baselga and Novoa } \\
(2000 \mathrm{~b})\end{array}$ & $\mathrm{F}$ & Eur \\
\hline $\begin{array}{l}\text { Entomoscelis adonidis } \\
\text { (Pallas) }\end{array}$ & 1974 & New record & $\mathrm{X}$ & WR \\
\hline $\begin{array}{l}\text { Galerucella lineola } \\
\text { (Fabricius) }\end{array}$ & 1993 & New record & $\mathrm{B}, \mathrm{C}, \mathrm{D}, \mathrm{E}, \mathrm{X}$ & WR \\
\hline $\begin{array}{l}\text { Galerucella calmariensis } \\
\text { (Linné) }\end{array}$ & 1993 & $\begin{array}{l}\text { Baselga and Novoa } \\
(2000 \mathrm{a})\end{array}$ & $A, B, G, X$ & WR \\
\hline
\end{tabular}


Appendix A Continued.

\begin{tabular}{|c|c|c|c|c|}
\hline Species & $\begin{array}{l}\text { First } \\
\text { record }\end{array}$ & Published records & Main areas & Dist. \\
\hline $\begin{array}{l}\text { Xanthogaleruca luteola } \\
\text { (Muller) }\end{array}$ & 1998 & $\begin{array}{l}\text { Baselga and Novoa } \\
(1999 a, 2000 b)\end{array}$ & $\mathrm{F}, \mathrm{G}, \mathrm{X}$ & WR \\
\hline $\begin{array}{l}\text { Galeruca macchoi } \\
\text { (Joannis) }\end{array}$ & 1981 & $\begin{array}{l}\text { Baselga and Novoa } \\
(2000 \mathrm{~b}, 2002 \mathrm{~b})\end{array}$ & $\mathrm{C}, \mathrm{F}, \mathrm{H}, \mathrm{X}$ & Ibe \\
\hline $\begin{array}{l}\text { Galeruca luctuosa } \\
\text { (Joannis) }\end{array}$ & 1934 & $\begin{array}{l}\text { Laboissière (1934) and } \\
\text { Baselga and Novoa } \\
\text { (1999a, 2002b) }\end{array}$ & $\mathrm{B}, \mathrm{G}, \mathrm{X}$ & Eur \\
\hline $\begin{array}{l}\text { Galeruca angusta } \\
\text { Küster }\end{array}$ & 1995 & $\begin{array}{l}\text { Baselga and Novoa } \\
(1999 a, 2000 a, c)\end{array}$ & $A, X$ & Med \\
\hline $\begin{array}{l}\text { Lochmaea scutellata } \\
\text { (Chevrolat) }\end{array}$ & 1934 & $\begin{array}{l}\text { Laboissière (1934) and } \\
\text { Baselga and Novoa } \\
\text { (1999b, 2000b, 2002b) }\end{array}$ & $\mathrm{B}, \mathrm{C}, \mathrm{E}, \mathrm{F}, \mathrm{H}, \mathrm{X}$ & Ibe \\
\hline $\begin{array}{l}\text { Lochmaea suturalis } \\
\text { (Thomson) }\end{array}$ & 1995 & $\begin{array}{l}\text { Baselga and Novoa } \\
(1999 b, 2000 a, b, 2002 b)\end{array}$ & $\mathrm{A}, \mathrm{C}, \mathrm{D}, \mathrm{E}, \mathrm{F}, \mathrm{H}, \mathrm{X}$ & Eur \\
\hline $\begin{array}{l}\text { Exosoma lusitanica } \\
\text { (Linné) }\end{array}$ & 1907 & $\begin{array}{l}\text { Chapman and Champion } \\
\text { (1907), Iglesias (1928) and } \\
\text { Baselga and Novoa } \\
(1999 a, 2000 a, b, 2002 b)\end{array}$ & $A, B, C, E, F, G, H, X, Y$ & WR \\
\hline $\begin{array}{l}\text { Calomicrus circumfusus } \\
\text { (Marsham) }\end{array}$ & 1866 & $\begin{array}{l}\text { López Seoane (1866), } \\
\text { Chapman and Champion } \\
\text { (1907) and Baselga and } \\
\text { Novoa (2000b, 2002b) }\end{array}$ & $\mathrm{B}, \mathrm{C}, \mathrm{D}, \mathrm{E}, \mathrm{F}, \mathrm{G}, \mathrm{H}, \mathrm{X}, \mathrm{Y}$ & Eur \\
\hline $\begin{array}{l}\text { Calomicrus suturalis } \\
\text { (Joannis) }\end{array}$ & 1998 & $\begin{array}{l}\text { Baselga and Novoa } \\
(2000 \mathrm{~b}, 2002 \mathrm{~b})\end{array}$ & $\mathrm{F}, \mathrm{G}$ & Eur \\
\hline $\begin{array}{l}\text { Luperus flavus } \\
\text { Rosenhauer }\end{array}$ & 1998 & $\begin{array}{l}\text { Baselga and Novoa } \\
(2000 \mathrm{~b}, 2002 \mathrm{~b})\end{array}$ & $\mathrm{E}, \mathrm{F}, \mathrm{G}, \mathrm{H}$ & Ibe \\
\hline $\begin{array}{l}\text { Luperus sulphuripes } \\
\text { Graëlls }\end{array}$ & 1866 & $\begin{array}{l}\text { López Seoane (1866) } \\
\text { and Baselga and } \\
\text { Novoa (1999a, 2000b, } \\
\text { 2002b) }\end{array}$ & $\mathrm{B}, \mathrm{C}, \mathrm{D}, \mathrm{E}, \mathrm{F}, \mathrm{G}, \mathrm{H}, \mathrm{X}, \mathrm{Y}$ & Ibe \\
\hline $\begin{array}{l}\text { Agelastica alni } \\
\text { (Linné) }\end{array}$ & 1928 & $\begin{array}{l}\text { Iglesias (1928) and } \\
\text { Baselga and Novoa } \\
\text { 1999a, 2000b, 2002b }\end{array}$ & $\mathrm{B}, \mathrm{E}, \mathrm{F}, \mathrm{G}, \mathrm{X}, \mathrm{Y}$ & WR \\
\hline $\begin{array}{l}\text { Sermylassa halensis } \\
\text { (Linné) }\end{array}$ & 1998 & $\begin{array}{l}\text { Baselga and Novoa } \\
(2000 \mathrm{~b})\end{array}$ & $\mathrm{F}, \mathrm{X}$ & Eur \\
\hline $\begin{array}{l}\text { Leptomona erythrocephala } \\
\text { (Olivier) }\end{array}$ & 1907 & $\begin{array}{l}\text { Heyden }(1870) \text { and } \\
\text { Baselga and Novoa } \\
(2000 \mathrm{a}, \mathrm{b})\end{array}$ & $\mathrm{A}, \mathrm{B}, \mathrm{C}, \mathrm{D}, \mathrm{F}, \mathrm{G}, \mathrm{X}, \mathrm{Y}$ & Med \\
\hline $\begin{array}{l}\text { Phyllotreta variipennis } \\
\text { (Boieldieu) }\end{array}$ & 1993 & $\begin{array}{l}\text { Baselga and Novoa } \\
\text { (2001a) }\end{array}$ & $\mathrm{B}, \mathrm{G}$ & Med \\
\hline $\begin{array}{l}\text { Phyllotreta vittula } \\
\text { (Redtenbacher) }\end{array}$ & 1993 & $\begin{array}{l}\text { Baselga and Novoa } \\
\text { (1999b) }\end{array}$ & $\mathrm{B}, \mathrm{X}$ & WR \\
\hline $\begin{array}{l}\text { Phyllotreta nemorum } \\
\text { (Linné) }\end{array}$ & 1993 & $\begin{array}{l}\text { Baselga and Novoa } \\
(2000 \mathrm{~b})\end{array}$ & $\mathrm{B}, \mathrm{C}, \mathrm{D}, \mathrm{E}, \mathrm{F}, \mathrm{X}$ & WR \\
\hline $\begin{array}{l}\text { Phyllotreta undulata } \\
\text { Kutschera }\end{array}$ & 1997 & $\begin{array}{l}\text { Baselga and Novoa } \\
(2000 \mathrm{a}, \mathrm{b})\end{array}$ & $\mathrm{A}, \mathrm{B}, \mathrm{C}, \mathrm{D}, \mathrm{F}, \mathrm{X}$ & WR \\
\hline
\end{tabular}


Appendix A Continued.

\begin{tabular}{|c|c|c|c|c|}
\hline Species & $\begin{array}{l}\text { First } \\
\text { record }\end{array}$ & Published records & Main areas & Dist. \\
\hline $\begin{array}{l}\text { Phyllotreta tetrastigma } \\
\text { (Comolli) }\end{array}$ & 1996 & $\begin{array}{l}\text { Baselga and Novoa } \\
(1998,2000 \mathrm{a}, \mathrm{b}, 2002 \mathrm{~b})\end{array}$ & $\mathrm{A}, \mathrm{B}, \mathrm{C}, \mathrm{D}, \mathrm{E}, \mathrm{F}, \mathrm{H}$ & WR \\
\hline $\begin{array}{l}\text { Phyllotreta striolata } \\
\text { (Fabricius) }\end{array}$ & 1870 & $\begin{array}{l}\text { Heyden (1870), González } \\
\text { de Andrés (1934) and } \\
\text { Baselga and Novoa } \\
(2000 \mathrm{a}, \mathrm{b})\end{array}$ & A, B, D, F, X, Y & WR \\
\hline $\begin{array}{l}\text { Phyllotreta ochripes } \\
\text { (Curtis) }\end{array}$ & 1993 & $\begin{array}{l}\text { Baselga and Novoa } \\
(2000 \mathrm{c})\end{array}$ & G & Eur \\
\hline $\begin{array}{l}\text { Phyllotreta exclamationis } \\
\text { (Thunberg) }\end{array}$ & 1997 & $\begin{array}{l}\text { Baselga and Novoa } \\
(1998,2000 \mathrm{a}, \mathrm{b})\end{array}$ & $\mathrm{A}, \mathrm{B}, \mathrm{C}, \mathrm{D}, \mathrm{E}, \mathrm{F}, \mathrm{X}$ & Eur \\
\hline $\begin{array}{l}\text { Phyllotreta atra } \\
\text { (Fabricius) }\end{array}$ & 1870 & $\begin{array}{l}\text { Heyden }(1870) \text { and } \\
\text { Baselga and Novoa } \\
(2000 \mathrm{a}, 2002 \mathrm{~b})\end{array}$ & $\mathrm{A}, \mathrm{B}, \mathrm{C}, \mathrm{D}, \mathrm{G}, \mathrm{H}, \mathrm{X}, \mathrm{Y}$ & WR \\
\hline $\begin{array}{l}\text { Phyllotreta cruciferae } \\
\text { (Goeze) }\end{array}$ & 1870 & $\begin{array}{l}\text { Heyden (1870), González } \\
\text { de Andrés (1934) and } \\
\text { Baselga and Novoa } \\
\text { (2000a) }\end{array}$ & $\mathrm{A}, \mathrm{B}, \mathrm{E}, \mathrm{X}, \mathrm{Y}$ & WR \\
\hline $\begin{array}{l}\text { Phyllotreta foudrasi } \\
\text { Brisout }\end{array}$ & 1993 & New record & $\mathrm{E}, \mathrm{G}$ & Med \\
\hline $\begin{array}{l}\text { Phyllotreta temperei } \\
\text { Doguet }\end{array}$ & 1993 & $\begin{array}{l}\text { Baselga and Novoa } \\
\text { (2001a) }\end{array}$ & $\mathrm{X}$ & Ibe \\
\hline $\begin{array}{l}\text { Phyllotreta consobrina } \\
\text { (Curtis) }\end{array}$ & 1993 & $\begin{array}{l}\text { Baselga and Novoa } \\
(1999 \mathrm{a}, 2000 \mathrm{a}, \mathrm{b}, 2002 \mathrm{~b})\end{array}$ & $\mathrm{A}, \mathrm{B}, \mathrm{C}, \mathrm{D}, \mathrm{E}, \mathrm{F}, \mathrm{G}, \mathrm{X}$ & WR \\
\hline $\begin{array}{l}\text { Phyllotreta nigripes } \\
\text { (Fabricius) }\end{array}$ & 1993 & $\begin{array}{l}\text { Baselga and Novoa } \\
(2000 \mathrm{~b}, 2002 \mathrm{~b})\end{array}$ & $\mathrm{B}, \mathrm{C}, \mathrm{D}, \mathrm{F}, \mathrm{H}, \mathrm{X}$ & WR \\
\hline $\begin{array}{l}\text { Phyllotreta ganglbaueri } \\
\text { Heikertinger }\end{array}$ & 2000 & $\begin{array}{l}\text { Baselga and Novoa } \\
\text { (2001b) }\end{array}$ & $\mathrm{X}$ & Eur \\
\hline $\begin{array}{l}\text { Phyllotreta procera } \\
\text { (Redtenbacher) }\end{array}$ & 1993 & $\begin{array}{l}\text { Baselga and Novoa } \\
\text { (2000a) }\end{array}$ & $\mathrm{A}, \mathrm{C}, \mathrm{G}, \mathrm{X}$ & WR \\
\hline $\begin{array}{l}\text { Aphthona lutescens } \\
\text { (Gyllenhal) }\end{array}$ & 1996 & $\begin{array}{l}\text { Baselga and Novoa } \\
(2000 \mathrm{a})\end{array}$ & $\mathrm{A}, \mathrm{B}, \mathrm{D}, \mathrm{X}$ & WR \\
\hline $\begin{array}{l}\text { Aphthona nigriceps } \\
\text { (Redtenbacher) }\end{array}$ & 1997 & $\begin{array}{l}\text { Baselga and Novoa } \\
\text { (2000a) }\end{array}$ & A, $X$ & WR \\
\hline $\begin{array}{l}\text { Aphthona punctiventris } \\
\text { Mulsant and Rey }\end{array}$ & 1993 & $\begin{array}{l}\text { Baselga and Novoa } \\
(1998,2000 \mathrm{a})\end{array}$ & $\mathrm{A}, \mathrm{C}, \mathrm{G}, \mathrm{X}$ & Med \\
\hline Aphthona occitana Doguet & 1997 & $\begin{array}{l}\text { Baselga and Novoa } \\
\text { (1999b) }\end{array}$ & B & Ibe \\
\hline $\begin{array}{l}\text { Aphthona atrocaerulea } \\
\text { (Stephens) }\end{array}$ & 1996 & $\begin{array}{l}\text { Baselga and Novoa } \\
(2000 \mathrm{a}, 2002 \mathrm{~b})\end{array}$ & $\mathrm{A}, \mathrm{E}, \mathrm{H}$ & WR \\
\hline $\begin{array}{l}\text { Aphthona melancholica } \\
\text { Weise }\end{array}$ & 1993 & $\begin{array}{l}\text { Baselga and Novoa } \\
(1999 b, 2000 \mathrm{~b}, \mathrm{c})\end{array}$ & $\mathrm{C}, \mathrm{F}, \mathrm{X}$ & Ibe \\
\hline $\begin{array}{l}\text { Aphthona sandrae } \\
\text { Baselga and Novoa }\end{array}$ & 1994 & $\begin{array}{l}\text { Baselga and Novoa } \\
\text { (2002a) }\end{array}$ & $C, X$ & Ibe \\
\hline $\begin{array}{l}\text { Aphthona nonstriata } \\
\text { (Goeze) }\end{array}$ & 1995 & $\begin{array}{l}\text { Baselga and Novoa } \\
(2000 \mathrm{a}, 2002 \mathrm{~b})\end{array}$ & $\mathrm{A}, \mathrm{B}, \mathrm{H}, \mathrm{X}$ & WR \\
\hline $\begin{array}{l}\text { Aphthona herbigrada } \\
\text { (Curtis) }\end{array}$ & 1997 & $\begin{array}{l}\text { Baselga and Novoa } \\
\text { (2001a) }\end{array}$ & $\mathrm{X}$ & WR \\
\hline $\begin{array}{l}\text { Longitarsus pellucidus } \\
\text { (Foudras) }\end{array}$ & 1994 & $\begin{array}{l}\text { Baselga and Novoa } \\
(2002 \mathrm{~b})\end{array}$ & G & WR \\
\hline
\end{tabular}


Appendix A Continued.

\begin{tabular}{|c|c|c|c|c|}
\hline Species & $\begin{array}{l}\text { First } \\
\text { record }\end{array}$ & Published records & Main areas & Dist. \\
\hline $\begin{array}{l}\text { Longitarsus codinai } \\
\text { Madar and Madar }\end{array}$ & 1996 & $\begin{array}{l}\text { Baselga and Novoa } \\
\text { (2000a) }\end{array}$ & A, $X$ & Med \\
\hline $\begin{array}{l}\text { Longitarsus ochroleucus } \\
\text { (Marsham) }\end{array}$ & 1997 & $\begin{array}{l}\text { Baselga and Novoa } \\
(2000 \mathrm{a}, 2002 \mathrm{~b})\end{array}$ & A, G, X & WR \\
\hline $\begin{array}{l}\text { Longitarsus candidulus } \\
\text { (Foudras) }\end{array}$ & 1997 & $\begin{array}{l}\text { Baselga and Novoa } \\
\text { (2000a) }\end{array}$ & A, G & Med \\
\hline $\begin{array}{l}\text { Longitarsus leonardii } \\
\text { Doguet }\end{array}$ & 2000 & $\begin{array}{l}\text { Baselga and Novoa } \\
\text { (2001a) }\end{array}$ & $\mathrm{X}$ & Ibe \\
\hline $\begin{array}{l}\text { Longitarsus flavicornis } \\
\text { (Stephens) }\end{array}$ & 1994 & $\begin{array}{l}\text { Baselga and Novoa } \\
(2000 \mathrm{a}, \mathrm{b}, 2002 \mathrm{~b})\end{array}$ & $\mathrm{A}, \mathrm{C}, \mathrm{D}, \mathrm{F}, \mathrm{G}, \mathrm{X}$ & WR \\
\hline $\begin{array}{l}\text { Longitarsus succineus } \\
\text { (Foudras) }\end{array}$ & 1991 & $\begin{array}{l}\text { Baselga and Novoa } \\
(2000 \mathrm{a})\end{array}$ & A, C, X & WR \\
\hline $\begin{array}{l}\text { Longitarsus aeruginosus } \\
\text { (Foudras) }\end{array}$ & 1996 & $\begin{array}{l}\text { Baselga and Novoa } \\
(2000 \mathrm{a}, \mathrm{b})\end{array}$ & $\mathrm{A}, \mathrm{B}, \mathrm{C}, \mathrm{F}, \mathrm{X}$ & WR \\
\hline $\begin{array}{l}\text { Longitarsus rubiginosus } \\
\text { (Foudras) }\end{array}$ & 1997 & $\begin{array}{l}\text { Baselga and Novoa } \\
\text { (1999b) }\end{array}$ & $\mathrm{X}$ & Eur \\
\hline $\begin{array}{l}\text { Longitarsus tabidus } \\
\text { (Fabricius) }\end{array}$ & 1992 & $\begin{array}{l}\text { Baselga and Novoa } \\
(2000 \mathrm{a}, \mathrm{b}, 2002 \mathrm{~b})\end{array}$ & $\mathrm{A}, \mathrm{B}, \mathrm{E}, \mathrm{F}, \mathrm{G}, \mathrm{X}$ & WR \\
\hline $\begin{array}{l}\text { Longitarsus australis } \\
\text { (Mulsant and Rey) }\end{array}$ & 1997 & $\begin{array}{l}\text { Baselga and Novoa } \\
(1998,2000 \mathrm{a})\end{array}$ & $A, X$ & Med \\
\hline $\begin{array}{l}\text { Longitarsus foudrasi } \\
\text { Weise }\end{array}$ & 2000 & $\begin{array}{l}\text { Baselga and Novoa } \\
(2001 \mathrm{a}, 2002 \mathrm{~b})\end{array}$ & G & WR \\
\hline $\begin{array}{l}\text { Longitarsus nigrofasciatus } \\
\text { (Goeze) }\end{array}$ & 1992 & $\begin{array}{l}\text { Baselga and Novoa } \\
(2000 \mathrm{a}, \mathrm{b}, 2002 \mathrm{~b})\end{array}$ & $\mathrm{A}, \mathrm{B}, \mathrm{E}, \mathrm{F}, \mathrm{G}, \mathrm{H}, \mathrm{X}$ & WR \\
\hline $\begin{array}{l}\text { Longitarsus suturatus } \\
\text { (Foudras) }\end{array}$ & 1993 & $\begin{array}{l}\text { Baselga and Novoa } \\
(2000 \mathrm{a}, 2002 \mathrm{~b})\end{array}$ & $\mathrm{A}, \mathrm{E}, \mathrm{G}, \mathrm{H}, \mathrm{X}$ & Med \\
\hline $\begin{array}{l}\text { Longitarsus rutilus } \\
\text { (Illiger) }\end{array}$ & 1993 & $\begin{array}{l}\text { Baselga and Novoa } \\
(1999 b, 2000 a, b)\end{array}$ & $\mathrm{A}, \mathrm{B}, \mathrm{C}, \mathrm{E}, \mathrm{F}, \mathrm{G}, \mathrm{X}$ & WR \\
\hline $\begin{array}{l}\text { Longitarsus lycopi } \\
\text { (Foudras) }\end{array}$ & 1993 & $\begin{array}{l}\text { Baselga and Novoa } \\
\text { (2000a, b, 2002b) }\end{array}$ & $\mathrm{A}, \mathrm{B}, \mathrm{F}, \mathrm{G}, \mathrm{H}, \mathrm{X}$ & WR \\
\hline $\begin{array}{l}\text { Longitarsus ordinatus } \\
\text { (Foudras) }\end{array}$ & 1993 & $\begin{array}{l}\text { Baselga and Novoa } \\
(2000 \mathrm{~b}, 2002 \mathrm{~b})\end{array}$ & $\mathrm{E}, \mathrm{F}, \mathrm{G}, \mathrm{H}, \mathrm{X}$ & Med \\
\hline $\begin{array}{l}\text { Longitarsus ferrugineus } \\
\text { (Foudras) }\end{array}$ & 1993 & $\begin{array}{l}\text { Baselga and Novoa } \\
(2000 \mathrm{a}, \mathrm{b})\end{array}$ & $A, F, G, X$ & WR \\
\hline $\begin{array}{l}\text { Longitarsus albineus } \\
\text { (Foudras) }\end{array}$ & 1993 & $\begin{array}{l}\text { Baselga and Novoa } \\
\text { (2000b) }\end{array}$ & G & WR \\
\hline $\begin{array}{l}\text { Longitarsus membranaceus } \\
\text { (Foudras) }\end{array}$ & 1993 & $\begin{array}{l}\text { Baselga and Novoa } \\
(2000 a, b, 2002 b)\end{array}$ & $\mathrm{A}, \mathrm{C}, \mathrm{D}, \mathrm{E}, \mathrm{F}, \mathrm{G}, \mathrm{X}$ & WR \\
\hline $\begin{array}{l}\text { Longitarsus melanocephalus } \\
\text { (De Geer) }\end{array}$ & 1870 & $\begin{array}{l}\text { Heyden }(1870) \text { and } \\
\text { Baselga and Novoa } \\
(2000 \mathrm{a}, \mathrm{b}, 2002 \mathrm{~b})\end{array}$ & $\mathrm{A}, \mathrm{B}, \mathrm{C}, \mathrm{D}, \mathrm{E}, \mathrm{F}, \mathrm{G}, \mathrm{H}, \mathrm{X}, \mathrm{Y}$ & WR \\
\hline $\begin{array}{l}\text { Longitarsus kutscherae } \\
\text { (Rye) }\end{array}$ & 1997 & $\begin{array}{l}\text { Baselga and Novoa } \\
\text { (2001a) }\end{array}$ & $\mathrm{B}$ & WR \\
\hline $\begin{array}{l}\text { Longitarsus curtus } \\
\text { (Allard) }\end{array}$ & 1991 & $\begin{array}{l}\text { Baselga and Novoa } \\
(2000 \mathrm{c})\end{array}$ & $\mathrm{C}, \mathrm{G}, \mathrm{X}$ & Eur \\
\hline $\begin{array}{l}\text { Longitarsus exsoletus } \\
\text { (Linné) }\end{array}$ & 1997 & $\begin{array}{l}\text { Baselga and Novoa } \\
(2000 \mathrm{a}, \mathrm{b})\end{array}$ & $\mathrm{A}, \mathrm{B}, \mathrm{D}, \mathrm{F}, \mathrm{X}$ & WR \\
\hline
\end{tabular}


Appendix A Continued.

\begin{tabular}{|c|c|c|c|c|}
\hline Species & $\begin{array}{l}\text { First } \\
\text { record }\end{array}$ & Published records & Main areas & Dist. \\
\hline $\begin{array}{l}\text { Longitarsus cerinthes } \\
\text { (Schrank) }\end{array}$ & 1997 & $\begin{array}{l}\text { Baselga and Novoa } \\
\text { (2000a, b, 2002b) }\end{array}$ & $\mathrm{A}, \mathrm{B}, \mathrm{F}, \mathrm{G}, \mathrm{X}$ & WR \\
\hline $\begin{array}{l}\text { Longitarsus scutellaris } \\
\text { (Mulsant and Rey) }\end{array}$ & 1993 & New record & $\mathrm{X}$ & Eur \\
\hline $\begin{array}{l}\text { Longitarsus pratensis } \\
\text { (Panzer) }\end{array}$ & 1990 & $\begin{array}{l}\text { Leonardi and Doguet } \\
\text { (1990) and Baselga and } \\
\text { Novoa (2000a, b, 2002b) }\end{array}$ & $\mathrm{A}, \mathrm{B}, \mathrm{C}, \mathrm{D}, \mathrm{E}, \mathrm{F}, \mathrm{G}, \mathrm{H}, \mathrm{X}, \mathrm{Y}$ & I WR \\
\hline $\begin{array}{l}\text { Longitarsus reichei } \\
\text { (Allard) }\end{array}$ & 1870 & $\begin{array}{l}\text { Heyden (1870), Jolivet } \\
\text { (1953), Petitpierre } \\
\text { (1999) and Baselga } \\
\text { and Novoa (2000b) }\end{array}$ & $\mathrm{B}, \mathrm{F}, \mathrm{X}, \mathrm{Y}$ & Eur \\
\hline $\begin{array}{l}\text { Longitarsus ballotae } \\
\text { (Marsham) }\end{array}$ & 1870 & Heyden (1870) & $\mathrm{G}, \mathrm{Y}$ & WR \\
\hline $\begin{array}{l}\text { Longitarsus gracilis } \\
\text { Kutschera }\end{array}$ & 1993 & $\begin{array}{l}\text { Baselga and Novoa } \\
(2000 a, b, 2002 b)\end{array}$ & $\mathrm{A}, \mathrm{B}, \mathrm{F}, \mathrm{H}, \mathrm{X}$ & WR \\
\hline $\begin{array}{l}\text { Longitarsus atricillus } \\
\text { (Linné) }\end{array}$ & 1991 & $\begin{array}{l}\text { Baselga and Novoa } \\
(2000 \mathrm{~b}, 2002 \mathrm{~b})\end{array}$ & $\mathrm{B}, \mathrm{C}, \mathrm{D}, \mathrm{E}, \mathrm{F}, \mathrm{G}, \mathrm{X}$ & WR \\
\hline $\begin{array}{l}\text { Longitarsus bedeli } \\
\text { Uhagón }\end{array}$ & 1993 & $\begin{array}{l}\text { Baselga and Novoa } \\
(2002 b)\end{array}$ & $\mathrm{H}$ & Ibe \\
\hline $\begin{array}{l}\text { Longitarsus lateripunctatus } \\
\text { Rosenhauer }\end{array}$ & 1993 & $\begin{array}{l}\text { Baselga and Novoa } \\
(2000 \mathrm{a}, \mathrm{b})\end{array}$ & $\mathrm{A}, \mathrm{B}, \mathrm{F}, \mathrm{G}, \mathrm{X}$ & WR \\
\hline $\begin{array}{l}\text { Longitarsus dorsalis } \\
\text { (Fabricius) }\end{array}$ & 1993 & $\begin{array}{l}\text { Baselga and Novoa } \\
(2000 \mathrm{~b}, 2002 \mathrm{~b})\end{array}$ & $\mathrm{F}, \mathrm{G}, \mathrm{H}, \mathrm{X}$ & WR \\
\hline $\begin{array}{l}\text { Longitarsus luridus } \\
\text { (Scopoli) }\end{array}$ & 1991 & $\begin{array}{l}\text { Baselga and Novoa } \\
(2000 a, b, 2002 b)\end{array}$ & $\mathrm{A}, \mathrm{B}, \mathrm{C}, \mathrm{D}, \mathrm{E}, \mathrm{F}, \mathrm{G}, \mathrm{H}, \mathrm{X}$ & WR \\
\hline $\begin{array}{l}\text { Longitarsus fulgens } \\
\text { (Foudras) }\end{array}$ & 2000 & $\begin{array}{l}\text { Baselga and Novoa } \\
\text { (2001a) }\end{array}$ & $\mathrm{C}$ & Eur \\
\hline $\begin{array}{l}\text { Longitarsus echii } \\
\text { (Koch) }\end{array}$ & 1993 & New record & E, $X$ & WR \\
\hline $\begin{array}{l}\text { Longitarsus aeneus } \\
\text { Kutschera }\end{array}$ & 1993 & $\begin{array}{l}\text { Baselga and Novoa } \\
(2000 \mathrm{a}, \mathrm{b})\end{array}$ & $\mathrm{A}, \mathrm{F}, \mathrm{X}$ & Med \\
\hline $\begin{array}{l}\text { Longitarsus niger } \\
\text { (Koch) }\end{array}$ & 1993 & New record & $\mathrm{X}$ & Eur \\
\hline $\begin{array}{l}\text { Longitarsus parvulus } \\
\text { (Paykull) }\end{array}$ & 1870 & $\begin{array}{l}\text { Heyden }(1870) \text { and } \\
\text { Baselga and Novoa } \\
(2000 \mathrm{a}, \mathrm{b})\end{array}$ & $\mathrm{A}, \mathrm{D}, \mathrm{F}, \mathrm{X}, \mathrm{Y}$ & WR \\
\hline $\begin{array}{l}\text { Longitarsus obliteratoides } \\
\text { Gruev }\end{array}$ & 1997 & $\begin{array}{l}\text { Baselga and Novoa } \\
(2002 b)\end{array}$ & G & Med \\
\hline $\begin{array}{l}\text { Longitarsus bergeali } \\
\text { Doguet and Gruev }\end{array}$ & 1998 & $\begin{array}{l}\text { Baselga and Novoa } \\
(2000 \mathrm{~b})\end{array}$ & $\mathrm{F}$ & Eur \\
\hline $\begin{array}{l}\text { Longitarsus anchusae } \\
\text { (Paykull) }\end{array}$ & 1993 & $\begin{array}{l}\text { Baselga and Novoa } \\
(2000 \mathrm{~b})\end{array}$ & E, F, X & WR \\
\hline $\begin{array}{l}\text { Altica ampelophaga } \\
\text { Guérin }\end{array}$ & 1981 & $\begin{array}{l}\text { Baselga and Novoa } \\
(1999 a, 2000 a, b, 2002 b)\end{array}$ & $\mathrm{A}, \mathrm{C}, \mathrm{D}, \mathrm{E}, \mathrm{F}, \mathrm{H}, \mathrm{X}$. & Med. \\
\hline $\begin{array}{l}\text { Altica brevicollis } \\
\text { Foudras }\end{array}$ & 1990 & $\begin{array}{l}\text { Baselga and Novoa } \\
(1999 \mathrm{~b}, 2000 \mathrm{~b})\end{array}$ & $\mathrm{C}, \mathrm{F}, \mathrm{X}$ & WR \\
\hline $\begin{array}{l}\text { Altica quercetorum } \\
\text { Foudras }\end{array}$ & 1993 & $\begin{array}{l}\text { Lombardero et al. } \\
\text { (1993) and Baselga } \\
\text { and Novoa (1999a) }\end{array}$ & $\mathrm{X}, \mathrm{Y}$ & Eur. \\
\hline
\end{tabular}


Appendix A Continued.

\begin{tabular}{|c|c|c|c|c|}
\hline Species & $\begin{array}{l}\text { First } \\
\text { record }\end{array}$ & Published records & Main areas & Dist. \\
\hline $\begin{array}{l}\text { Altica tamaricis } \\
\text { Schrank ssp. } \\
\text { franzi Král }\end{array}$ & 1966 & Král (1966) & $\mathrm{Y}$ & WR \\
\hline $\begin{array}{l}\text { Altica oleracea } \\
\text { (Linné) }\end{array}$ & 1981 & $\begin{array}{l}\text { Baselga and Novoa } \\
(2000 \mathrm{a}, \mathrm{b}, 2002 \mathrm{~b})\end{array}$ & $\mathrm{A}, \mathrm{B}, \mathrm{D}, \mathrm{E}, \mathrm{F}, \mathrm{G}, \mathrm{H}, \mathrm{X}$ & WR \\
\hline $\begin{array}{l}\text { Altica palustris } \\
\text { (Weise) }\end{array}$ & 1993 & $\begin{array}{l}\text { Baselga and Novoa } \\
(2000 \mathrm{~b})\end{array}$ & $\mathrm{C}, \mathrm{G}, \mathrm{X}$ & WR \\
\hline $\begin{array}{l}\text { Altica carduorum } \\
\text { Guérin }\end{array}$ & 1990 & $\begin{array}{l}\text { Baselga and Novoa } \\
(2000 \mathrm{c})\end{array}$ & $\mathrm{X}$. & WR. \\
\hline $\begin{array}{l}\text { Altica ericeti } \\
\text { (Allard) }\end{array}$ & 1981 & $\begin{array}{l}\text { Baselga and Novoa } \\
\text { (1999a, 2000b, 2002b) }\end{array}$ & $\mathrm{B}, \mathrm{C}, \mathrm{D}, \mathrm{E}, \mathrm{F}, \mathrm{G}, \mathrm{H}, \mathrm{X}$ & Eur \\
\hline $\begin{array}{l}\text { Altica longicollis } \\
\text { (Allard) }\end{array}$ & 1980 & $\begin{array}{l}\text { Baselga and Novoa } \\
\text { (1999b, 2000b) }\end{array}$ & $\mathrm{F}, \mathrm{X}$ & Eur \\
\hline $\begin{array}{l}\text { Hermaeophaga cicatrix } \\
\text { (Illiger) }\end{array}$ & 1999 & $\begin{array}{l}\text { Baselga and Novoa } \\
\text { (2001a) }\end{array}$ & $\mathrm{C}$ & WR \\
\hline $\begin{array}{l}\text { Batophila aerata } \\
\text { (Marsham) }\end{array}$ & 2000 & $\begin{array}{l}\text { Baselga and Novoa } \\
(2001 \mathrm{a}, 2002 \mathrm{~b})\end{array}$ & G & WR \\
\hline $\begin{array}{l}\text { Arrhenocoela lineata } \\
\text { (Rossi) }\end{array}$ & 1991 & $\begin{array}{l}\text { Baselga and Novoa } \\
(2000 \mathrm{~b}, 2002 \mathrm{~b})\end{array}$ & $\mathrm{C}, \mathrm{D}, \mathrm{F}, \mathrm{G}, \mathrm{X}$ & Med \\
\hline $\begin{array}{l}\text { Ochrosis ventralis } \\
\text { (Illiger) }\end{array}$ & 1997 & $\begin{array}{l}\text { Baselga and Novoa } \\
(2000 \mathrm{a}, \mathrm{b}, 2002 \mathrm{~b})\end{array}$ & $\mathrm{A}, \mathrm{F}, \mathrm{G}$ & WR \\
\hline $\begin{array}{l}\text { Neocrepidodera transversa } \\
\text { (Marsham) }\end{array}$ & 1997 & $\begin{array}{l}\text { Baselga and Novoa } \\
(2000 \mathrm{~b}, 2002 \mathrm{~b})\end{array}$ & $\mathrm{B}, \mathrm{E}, \mathrm{F}, \mathrm{H}, \mathrm{X}$ & Eur \\
\hline $\begin{array}{l}\text { Neocrepidodera impressa } \\
\text { (Fabricius) }\end{array}$ & 1976 & $\begin{array}{l}\text { Baselga and Novoa } \\
\text { (2000a) }\end{array}$ & $A, X$ & Med \\
\hline $\begin{array}{l}\text { Neocrepidodera ferruginea } \\
\text { (Scopoli) }\end{array}$ & 1996 & $\begin{array}{l}\text { Baselga and Novoa } \\
(1999 \mathrm{a}, 2000 \mathrm{~b})\end{array}$ & $\mathrm{B}, \mathrm{D}, \mathrm{F}, \mathrm{X}$ & WR \\
\hline $\begin{array}{l}\text { Neocrepidodera crassicornis } \\
\text { (Faldermann) ssp. } \\
\text { hispanica (J. Daniel) }\end{array}$ & 1996 & $\begin{array}{l}\text { Baselga and Novoa } \\
(2000 \mathrm{a}, \mathrm{b})\end{array}$ & $\mathrm{A}, \mathrm{B}, \mathrm{F}, \mathrm{X}$ & Med \\
\hline $\begin{array}{l}\text { Crepidodera fulvicornis } \\
\text { (Fabricius) }\end{array}$ & 1996 & $\begin{array}{l}\text { Baselga and Novoa } \\
(2000 \mathrm{a}, 2002 \mathrm{~b})\end{array}$ & $\mathrm{A}, \mathrm{B}, \mathrm{C}, \mathrm{D}, \mathrm{E}, \mathrm{H}, \mathrm{X}$ & WR \\
\hline $\begin{array}{l}\text { Crepidodera aureola } \\
\text { (Foudras) }\end{array}$ & 1993 & $\begin{array}{l}\text { Baselga and Novoa } \\
(2000 \mathrm{a}, \mathrm{b}, 2002 \mathrm{~b})\end{array}$ & $\mathrm{A}, \mathrm{B}, \mathrm{C}, \mathrm{D}, \mathrm{E}, \mathrm{F}, \mathrm{G}, \mathrm{H}, \mathrm{X}$ & Med \\
\hline $\begin{array}{l}\text { Crepidodera aurata } \\
\text { (Marsham) }\end{array}$ & 1981 & $\begin{array}{l}\text { Baselga and Novoa } \\
(2000 \mathrm{~b}, 2002 \mathrm{~b})\end{array}$ & $\mathrm{F}, \mathrm{G}, \mathrm{X}$ & WR \\
\hline $\begin{array}{l}\text { Epitrix pubescens } \\
\text { (Koch) }\end{array}$ & 1907 & $\begin{array}{l}\text { Chapman and Champion } \\
\text { (1907) and Baselga and } \\
\text { Novoa }(2000 \mathrm{a}, \mathrm{b})\end{array}$ & $\mathrm{A}, \mathrm{F}, \mathrm{X}, \mathrm{Y}$ & WR \\
\hline $\begin{array}{l}\text { Epitrix intermedia } \\
\text { Foudras }\end{array}$ & 1996 & $\begin{array}{l}\text { Baselga and Novoa } \\
(1999 \mathrm{~b}, 2000 \mathrm{~b})\end{array}$ & $\mathrm{F}, \mathrm{X}$ & Eur \\
\hline $\begin{array}{l}\text { Podagrica fuscicornis } \\
\text { (Linné) }\end{array}$ & 1997 & New record & $\mathrm{B}, \mathrm{X}$ & WR \\
\hline $\begin{array}{l}\text { Podagrica fuscipes } \\
\text { (Fabricius) }\end{array}$ & 1993 & $\begin{array}{l}\text { Baselga and Novoa } \\
(2000 \mathrm{a}, \mathrm{b}, 2002 \mathrm{~b})\end{array}$ & $\mathrm{A}, \mathrm{B}, \mathrm{C}, \mathrm{E}, \mathrm{F}, \mathrm{G}, \mathrm{H}, \mathrm{X}$ & Eur \\
\hline $\begin{array}{l}\text { Mantura chrysanthemi } \\
\text { (Koch) }\end{array}$ & 1993 & New record & $\mathrm{X}$ & WR \\
\hline
\end{tabular}


Appendix A Continued.

\begin{tabular}{|c|c|c|c|c|}
\hline Species & $\begin{array}{l}\text { First } \\
\text { record }\end{array}$ & Published records & Main areas & Dist. \\
\hline $\begin{array}{l}\text { Mantura lutea } \\
\text { (Allard) }\end{array}$ & 1998 & $\begin{array}{l}\text { Baselga and Novoa } \\
\text { (2000a) }\end{array}$ & A & Med \\
\hline $\begin{array}{l}\text { Mantura rustica } \\
\text { (Linné) }\end{array}$ & 1997 & $\begin{array}{l}\text { Baselga and Novoa } \\
\text { (2000a) }\end{array}$ & A, D & WR \\
\hline $\begin{array}{l}\text { Chaetocnema chlorophana } \\
\text { (Duftschmid) }\end{array}$ & 1993 & $\begin{array}{l}\text { Baselga and Novoa } \\
(2000 \mathrm{a}, 2002 \mathrm{~b})\end{array}$ & $A, E, G, X$ & WR \\
\hline $\begin{array}{l}\text { Chaetocnema concinna } \\
\text { (Marsham) }\end{array}$ & 1992 & $\begin{array}{l}\text { Baselga and Novoa } \\
(1999 b, 2000 a)\end{array}$ & $\mathrm{A}, \mathrm{B}, \mathrm{D}, \mathrm{X}$ & WR \\
\hline $\begin{array}{l}\text { Chaetocnema laevicollis } \\
\text { (Thomson) }\end{array}$ & 1992 & $\begin{array}{l}\text { Baselga and Novoa } \\
(1999 \mathrm{~b}, 2000 \mathrm{a}, \mathrm{b})\end{array}$ & $\mathrm{A}, \mathrm{B}, \mathrm{C}, \mathrm{E}, \mathrm{F}, \mathrm{X}$ & WR \\
\hline $\begin{array}{l}\text { Chaetocnema tibialis } \\
\text { (Illiger) }\end{array}$ & 1993 & $\begin{array}{l}\text { Baselga and Novoa } \\
(2000 \mathrm{a}, \mathrm{b}, 2002 \mathrm{~b})\end{array}$ & $\mathrm{A}, \mathrm{E}, \mathrm{F}, \mathrm{G}, \mathrm{X}$ & WR \\
\hline $\begin{array}{l}\text { Chaetocnema depressa } \\
\text { (Boieldieu) }\end{array}$ & 2000 & $\begin{array}{l}\text { Baselga and Novoa } \\
(2001 \mathrm{a}, 2002 \mathrm{~b})\end{array}$ & G & Med \\
\hline $\begin{array}{l}\text { Chaetocnema aridula } \\
\text { (Gyllenhal) }\end{array}$ & 1996 & $\begin{array}{l}\text { Baselga and Novoa } \\
(2000 \mathrm{~b})\end{array}$ & $\mathrm{D}, \mathrm{F}, \mathrm{X}$ & WR \\
\hline $\begin{array}{l}\text { Chaetocnema confusa } \\
\text { (Boheman) }\end{array}$ & 1996 & $\begin{array}{l}\text { Baselga and Novoa } \\
(2000 \mathrm{c}, 2002 \mathrm{~b})\end{array}$ & $\mathrm{H}, \mathrm{X}$ & Eur \\
\hline $\begin{array}{l}\text { Chaetocnema arida } \\
\text { Foudras }\end{array}$ & 1991 & $\begin{array}{l}\text { Baselga and Novoa } \\
(2000 \mathrm{a}, \mathrm{b}, 2002 \mathrm{~b})\end{array}$ & $\mathrm{A}, \mathrm{B}, \mathrm{C}, \mathrm{D}, \mathrm{E}, \mathrm{F}, \mathrm{G}, \mathrm{H}, \mathrm{X}$ & WR \\
\hline $\begin{array}{l}\text { Chaetocnema paganettii } \\
\text { Heikertinger }\end{array}$ & 1964 & $\begin{array}{l}\text { Baselga and Novoa } \\
(2002 b)\end{array}$ & $\mathrm{H}$ & Med \\
\hline $\begin{array}{l}\text { Chaetocnema hortensis } \\
\text { (Geoffroy) }\end{array}$ & 1870 & $\begin{array}{l}\text { Heyden (1870) and } \\
\text { Baselga and Novoa } \\
(2000 \mathrm{a}, \mathrm{b}, 2002 \mathrm{~b})\end{array}$ & $\mathrm{A}, \mathrm{B}, \mathrm{C}, \mathrm{D}, \mathrm{E}, \mathrm{F}, \mathrm{H}, \mathrm{X}, \mathrm{Y}$ & WR \\
\hline $\begin{array}{l}\text { Oedionychus cinctus } \\
\text { (Fabricius) }\end{array}$ & 1928 & $\begin{array}{l}\text { Iglesias (1928), } \\
\text { Biondi (1991), } \\
\text { Bastazo et al. } \\
\text { (1993) and Baselga } \\
\text { and Novoa (1998, } \\
\text { 1999a, 2000a, b, 2002b) }\end{array}$ & $\mathrm{A}, \mathrm{B}, \mathrm{C}, \mathrm{D}, \mathrm{E}, \mathrm{F}, \mathrm{G}, \mathrm{H}, \mathrm{X}, \mathrm{Y}$ & Ibe \\
\hline $\begin{array}{l}\text { Sphaeroderma testaceum } \\
\text { (Fabricius) }\end{array}$ & 1990 & $\begin{array}{l}\text { Baselga and Novoa } \\
(2000 \mathrm{~b})\end{array}$ & $\mathrm{C}, \mathrm{D}, \mathrm{F}, \mathrm{X}$ & Eur \\
\hline $\begin{array}{l}\text { Sphaeroderma rubidum } \\
\text { (Graëlls) }\end{array}$ & 1991 & $\begin{array}{l}\text { Baselga and Novoa } \\
(2000 \mathrm{a}, \mathrm{b}, 2002 \mathrm{~b})\end{array}$ & $\mathrm{A}, \mathrm{C}, \mathrm{F}, \mathrm{G}, \mathrm{H}, \mathrm{X}$ & WR \\
\hline $\begin{array}{l}\text { Apteropeda globosa } \\
\text { (Illiger) }\end{array}$ & 1991 & $\begin{array}{l}\text { Baselga and Novoa } \\
(1999 \mathrm{~b}, 2000 \mathrm{~b})\end{array}$ & $\mathrm{C}, \mathrm{F}, \mathrm{X}$ & Eur \\
\hline $\begin{array}{l}\text { Apteropeda orbiculata } \\
\text { (Marsham) }\end{array}$ & 1991 & $\begin{array}{l}\text { Biondi (1991) and } \\
\text { Baselga and Novoa } \\
(2000 \mathrm{a}, \mathrm{b})\end{array}$ & $\mathrm{A}, \mathrm{B}, \mathrm{C}, \mathrm{D}, \mathrm{F}, \mathrm{X}, \mathrm{Y}$ & Eur \\
\hline $\begin{array}{l}\text { Apteropeda ovulum } \\
\text { (Illiger) }\end{array}$ & 1996 & $\begin{array}{l}\text { Baselga and Novoa } \\
\text { 1999a }\end{array}$ & $\mathrm{D}, \mathrm{X}$ & Med \\
\hline $\begin{array}{l}\text { Dibolia occultans } \\
\text { (Koch) }\end{array}$ & 1992 & $\begin{array}{l}\text { Baselga and Novoa } \\
(2000 a, b, 2002 b)\end{array}$ & $\mathrm{A}, \mathrm{D}, \mathrm{E}, \mathrm{F}, \mathrm{H}, \mathrm{X}$ & WR \\
\hline $\begin{array}{l}\text { Psylliodes affinis } \\
\text { (Paykull) }\end{array}$ & 1993 & $\begin{array}{l}\text { Baselga and Novoa } \\
\text { (1999b, 2000b, 2002b) }\end{array}$ & $\mathrm{B}, \mathrm{C}, \mathrm{D}, \mathrm{F}, \mathrm{G}, \mathrm{X}$ & WR \\
\hline $\begin{array}{l}\text { Psylliodes marcidus } \\
\text { (Illiger) }\end{array}$ & 1997 & $\begin{array}{l}\text { Baselga and Novoa } \\
\text { (2000a) }\end{array}$ & A & WR \\
\hline
\end{tabular}


Appendix A Continued.

\begin{tabular}{|c|c|c|c|c|}
\hline Species & $\begin{array}{l}\text { First } \\
\text { record }\end{array}$ & Published records & Main areas & Dist. \\
\hline $\begin{array}{l}\text { Psylliodes pallidipennis } \\
\text { Rosenhauer }\end{array}$ & 1997 & $\begin{array}{l}\text { Baselga and Novoa } \\
\text { (2000a) }\end{array}$ & A & Med \\
\hline $\begin{array}{l}\text { Psylliodes chrysocephalus } \\
\text { (Linné) }\end{array}$ & 1993 & $\begin{array}{l}\text { Baselga and Novoa } \\
(2000 \mathrm{a}, \mathrm{b})\end{array}$ & $\mathrm{A}, \mathrm{B}, \mathrm{C}, \mathrm{E}, \mathrm{F}, \mathrm{X}$ & WR \\
\hline $\begin{array}{l}\text { Psylliodes napi } \\
\text { (Fabricius) }\end{array}$ & 1934 & $\begin{array}{l}\text { González de Andrés } \\
(1934) \text { and Baselga } \\
\text { and Novoa }(2000 \mathrm{a}, \mathrm{b})\end{array}$ & $\mathrm{C}, \mathrm{F}, \mathrm{X}, \mathrm{Y}$ & WR \\
\hline $\begin{array}{l}\text { Psylliodes laticollis } \\
\text { Kutschera }\end{array}$ & 1997 & $\begin{array}{l}\text { Baselga and Novoa } \\
(2000 \mathrm{a})\end{array}$ & A, D & WR \\
\hline $\begin{array}{l}\text { Psylliodes toelgi } \\
\text { Heikertinger }\end{array}$ & 1997 & $\begin{array}{l}\text { Baselga and Novoa } \\
(2002 \mathrm{~b})\end{array}$ & G & Eur \\
\hline $\begin{array}{l}\text { Psylliodes cupreus } \\
\text { (Koch) }\end{array}$ & 1993 & $\begin{array}{l}\text { Baselga and Novoa } \\
(2000 a, b, 2002 b)\end{array}$ & $\mathrm{A}, \mathrm{B}, \mathrm{E}, \mathrm{F}, \mathrm{H}, \mathrm{X}$ & WR \\
\hline $\begin{array}{l}\text { Psylliodes fusiformis } \\
\text { (Illiger) }\end{array}$ & 1997 & $\begin{array}{l}\text { Baselga and Novoa } \\
(2000 \mathrm{a})\end{array}$ & A & Med \\
\hline $\begin{array}{l}\text { Psylliodes vindobonensis } \\
\text { Heikertinger }\end{array}$ & 1982 & $\begin{array}{l}\text { Baselga and Novoa } \\
(2002 b)\end{array}$ & $\mathrm{H}$ & Eur \\
\hline $\begin{array}{l}\text { Psylliodes hispanus } \\
\text { Heikertinger }\end{array}$ & 1993 & $\begin{array}{l}\text { Baselga and Novoa } \\
\text { (2001a) }\end{array}$ & $\mathrm{C}, \mathrm{X}$ & Ibe \\
\hline $\begin{array}{l}\text { Psylliodes dulcamarae } \\
\text { (Koch) }\end{array}$ & 1994 & $\begin{array}{l}\text { Baselga and Novoa } \\
(2001 \mathrm{a}, 2002 \mathrm{~b})\end{array}$ & $\mathrm{G}, \mathrm{X}$ & WR \\
\hline $\begin{array}{l}\text { Psylliodes cucullatus } \\
\text { (Illiger) ssp. } \\
\text { heydeni Weise }\end{array}$ & 1991 & $\begin{array}{l}\text { Baselga and Novoa } \\
(1999 b, 2000 a, b)\end{array}$ & $\mathrm{A}, \mathrm{B}, \mathrm{C}, \mathrm{D}, \mathrm{F}, \mathrm{X}$ & WR \\
\hline $\begin{array}{l}\text { Psylliodes gougeleti } \\
\text { All. }\end{array}$ & 1866 & $\begin{array}{l}\text { López Seoane (1866, } \\
1877) \text { and Baselga and } \\
\text { Novoa (2000a, b, 2002b) }\end{array}$ & $\mathrm{A}, \mathrm{B}, \mathrm{C}, \mathrm{D}, \mathrm{E}, \mathrm{F}, \mathrm{H}, \mathrm{X}, \mathrm{Y}$ & Med \\
\hline $\begin{array}{l}\text { Psylliodes cervinoi } \\
\text { Baselga and Novoa }\end{array}$ & 1993 & $\begin{array}{l}\text { Baselga and Novoa } \\
(2003)\end{array}$ & $\mathrm{H}$ & Ibe \\
\hline $\begin{array}{l}\text { Dicladispa testacea } \\
\text { (Linné) }\end{array}$ & 1907 & $\begin{array}{l}\text { Chapman and Champion } \\
(1907) \text { and Baselga and } \\
\text { Novoa (1999a, 2000a, b, } \\
\text { 2002b) }\end{array}$ & A, B, F, G, X, Y & Med \\
\hline $\begin{array}{l}\text { Hispa atra } \\
\text { Linné }\end{array}$ & 1907 & $\begin{array}{l}\text { Chapman and Champion } \\
\text { (1907) and Baselga and } \\
\text { Novoa }(2000 \mathrm{~b}, 2002 \mathrm{~b})\end{array}$ & B, C, D, E, F, H, X, Y & WR \\
\hline $\begin{array}{l}\text { Cassida viridis } \\
\text { Linné }\end{array}$ & 1993 & $\begin{array}{l}\text { Baselga and Novoa } \\
(1999 \mathrm{a}, 2000 \mathrm{a}, 2002 \mathrm{~b})\end{array}$ & $\mathrm{A}, \mathrm{B}, \mathrm{D}, \mathrm{E}, \mathrm{G}, \mathrm{H}, \mathrm{X}$ & WR \\
\hline $\begin{array}{l}\text { Cassida hemisphaerica } \\
\text { Herbst }\end{array}$ & 1997 & $\begin{array}{l}\text { Baselga and Novoa } \\
(2000 \mathrm{a}, \mathrm{b}, 2002 \mathrm{~b})\end{array}$ & $\mathrm{A}, \mathrm{F}, \mathrm{H}, \mathrm{X}$ & WR \\
\hline $\begin{array}{l}\text { Cassida nebulosa } \\
\text { Linné }\end{array}$ & 1998 & $\begin{array}{l}\text { Baselga and Novoa } \\
(2000 \mathrm{~b})\end{array}$ & F & WR \\
\hline $\begin{array}{l}\text { Cassida flaveola } \\
\text { Thunberg }\end{array}$ & 1993 & $\begin{array}{l}\text { Baselga and Novoa } \\
(1999 b, 2000 a, b)\end{array}$ & $\mathrm{A}, \mathrm{B}, \mathrm{F}, \mathrm{X}$ & WR \\
\hline $\begin{array}{l}\text { Cassida seladonia } \\
\text { Gyllenhal }\end{array}$ & 1991 & $\begin{array}{l}\text { Baselga and Novoa } \\
(2000 \mathrm{~b})\end{array}$ & $\mathrm{B}, \mathrm{F}, \mathrm{X}$ & WR \\
\hline
\end{tabular}


Appendix A Continued.

\begin{tabular}{|c|c|c|c|c|}
\hline Species & $\begin{array}{l}\text { First } \\
\text { record }\end{array}$ & Published records & Main areas & Dist. \\
\hline $\begin{array}{l}\text { Cassida vibex } \\
\text { Linné }\end{array}$ & 1991 & $\begin{array}{l}\text { Baselga and Novoa } \\
\text { (2000b) }\end{array}$ & $\mathrm{B}, \mathrm{D}, \mathrm{F}, \mathrm{X}$ & WR \\
\hline $\begin{array}{l}\text { Cassida rubiginosa } \\
\text { Müller }\end{array}$ & 1990 & $\begin{array}{l}\text { Baselga and Novoa } \\
(2000 \mathrm{a}, \mathrm{b}, 2002 \mathrm{~b})\end{array}$ & $\mathrm{A}, \mathrm{B}, \mathrm{C}, \mathrm{D}, \mathrm{E}, \mathrm{F}, \mathrm{H}$ & WR \\
\hline $\begin{array}{l}\text { Cassida inquinata } \\
\text { Brullé }\end{array}$ & 1866 & $\begin{array}{l}\text { López Seoane (1866) } \\
\text { and Baselga and } \\
\text { Novoa (2000a) }\end{array}$ & $\mathrm{B}, \mathrm{Y}$ & WR \\
\hline $\begin{array}{l}\text { Cassida hexastigma } \\
\text { Suffrian }\end{array}$ & 1997 & Bordy (2000) & $\mathrm{A}, \mathrm{G}$ & Med \\
\hline $\begin{array}{l}\text { Cassida denticollis } \\
\text { Suffrian }\end{array}$ & 1993 & $\begin{array}{l}\text { Baselga and Novoa } \\
\text { (1999b) }\end{array}$ & $\mathrm{B}, \mathrm{C}, \mathrm{D}, \mathrm{X}$ & WR \\
\hline $\begin{array}{l}\text { Cassida subreticulata } \\
\text { Suffrian }\end{array}$ & 2000 & $\begin{array}{l}\text { Baselga and Novoa } \\
(1999 a, 2001 a)\end{array}$ & $\mathrm{D}, \mathrm{X}$ & WR \\
\hline $\begin{array}{l}\text { Cassida vittata } \\
\text { Villers }\end{array}$ & 1997 & $\begin{array}{l}\text { Baselga and Novoa } \\
\text { (2000a) }\end{array}$ & A, B & WR \\
\hline
\end{tabular}

\section{References}

Báguena L. 1959. Cuatro novedades y un comentario sobre Coleopteros de España. Eos 35: 209-214.

Báguena L. 1960a. Los Donaciinae ibéricos de la Colección del Instituto Español de Entomología. Graellsia 18: 3-14.

Báguena L. 1960b. Clave práctica para la determinación de los Clytrini ibéricos. Graellsia 18: $123-145$.

Báguena L. 1960c. Notas críticas y descriptivas sobre algunos Clytrini. Graellsia 18: 15-28.

Baselga A. and Novoa F. 1998. Citas nuevas o interesantes de Chrysomelidae (Coleoptera) del Noroeste peninsular. Bol. Asoc. Española Entomol. 22(3-4): 246-248.

Baselga A. and Novoa F. 1999a. La colección de Chrysomelidae (Coleoptera) del Museo de Historia Natural Luis Iglesias (Universidad de Santiago de Compostela, España). Nova Acta Científica Compostelana (Bioloxía) 9: 307-312.

Baselga A. and Novoa F. 1999b. Citas interesantes de Alticinae (Coleoptera, Chrysomelidae) de la Península Ibérica. Bol. Asoc. Española Entomol. 23(12): 53-57.

Baselga A. and Novoa F. 2000a. Los Chrysomelidae (Coleoptera) de los sistemas dunares de Galicia (Noroeste de España). Bol. Real Soc. Española Hist. Nat. (Sec. Biol.) 96(12): 113-124. Baselga A. and Novoa F. 2000b. Los Chrysomelidae (Coleoptera) de la Sierra de Ancares, Noroeste de la España (Coleoptera). Nouvelle Rev. d'Entomol. (N.S.) 17(2): 165-180.

Baselga A. and Novoa F. 2000c. Citas nuevas o interesantes de Chrysomelidae (Coleoptera) del Norte de la Península Ibérica. Bol. Asoc. Española Entomol. 24(3-4): 15-22.

Baselga A. and Novoa F. 2000d. Crypytocephalus cantabricus Franz, a poorly known endemic species from the northwest of the Iberian Peninsula (Coleoptera: Chrysomelidae). Koleopterologische Rundschau 70: 191-195.

Baselga A. and Novoa F. 2001a. Citas nuevas de Chrysomelidae (Coleoptera) de Galicia (Noroeste de la Península Ibérica). Bol. Asoc. Española Entomol. 25(1-2): 125-131.

Baselga A. and Novoa F. 2001b. Phyllotreta ganglbaueri Heikertinger, 1909, especie nueva para la Península Ibérica (Coleoptera, Chrysomelidae). Nouvelle Rev. d’Entomol. (N.S.) 18(1): 89-90. 
Baselga A. and Novoa F. 2002a. New species of Aphthona (Coleoptera: Chrysomelidae: Alticinae) and key to Iberian species of Aphthona hammarstroemi group. Can. Entomol. 134(1): 1-7.

Baselga A. and Novoa F. 2002b. Los Chrysomelidae (Coleoptera) de las sierras orientales de Ourense (Galicia, noroeste de la Península Ibérica). Bol. Asoc. Española Entomol. 26(3-4): 5773.

Baselga A. and Novoa F. 2003. A new species of Psylliodes (Coleoptera: Chrysomelidae) and key to the wingless species from the Iberian Peninsula. Ann. Entomol. Soc. Am. 96(6): 689-692.

Baselga A. and Novoa F. 2004. Coleópteros del Parque Natural de las Fragas del Eume (Galicia, noroeste de la Península Ibérica), II: Scarabaeoidea, Buprestoidea, Byrrhoidea, Elateroidea, Bostrichoidea, Lymexyloidea, Cleroidea, Cucujoidea, Tenebrionoidea, Chrysomeloidea y Curculionoidea. Bol. Asoc. Española Entomol. 28(1-2): 121-143.

Bastazo G., Vela J.M. and Petitpierre E. 1993. Datos faunísticos sobre Alticinae ibéricos. Bol. Asoc. Española Entomol. 17(1): 45-69.

Bechyné J. 1948. Contribution à la connaiscence du genre Timarcha Latr. 12: Etudes phylogenetiques et zoogeographiques (Col. Phyt. Chrys.). Sborn. Narod. Mus. Praze 4B: 1-62.

Bechyné J. 1957. Notes sur le Phytodecta (Spartoxena) variabilis Oliv. de l'Espagne (Col. Phytophaga). Eos 33: 263-267.

Bedel L. 1901. Faune des Coléoptères du Bassin de la Seine, V. Phythophaga. Société Entomologique de France, Paris.

Biondi M. 1991. Note faunistiche, tassonomiche ed ecologiche su alcune specie di Chrysomelidae Alticinae della Penisolla Iberica (Col.). Eos 66(2): 161-172.

Bordy B. 2000. Coléoptères Chrysomelidae. Vol. 3, Cassidinae. Faune de France 80. Federation Française des Societés de Sciences Naturelles, Paris.

Bourdonné J.C. 1994. Les Cryptocephalus rouges ouest méditerranéens du groupe de C. bimaculatus Fabricius (Coleoptera, Chrysomelidae, Cryptocephalinae). Nouvelle Rev. d'Entomol. (N.S.) 11(2): 185-209.

Burlini M. 1955. Revisione dei Cryptocephalus Italiani e della maggior parte specie di Europa. Mem. Soc. Entomol. Ital. 34: 5-287.

Cabrero-Sañudo F.J. and Lobo L.M. 2003. Estimating the number of species not yet described and their characteristics: the case of Western Palaearctic dung beetle species (Coleoptera, Scarabaeoidea). Biodiv. Conserv. 12: 147-166.

Carlton C.E. and Robison H.W. 1998. Diversity of litter-dwelling beetles in the Ouachita Highlands of Arkansas, USA (Insecta: Coleoptera). Biodiv. Conserv. 7: 1589-1605.

Chapman T.A. and Champion G.C. 1907. Entomology in NW Spain (Galicia and León). Trans. Ent. Soc. London 1907: 147-171.

Cobos A. 1954. Nota sobre el género Cyrtonus Latr. (Coleoptera Chrysomelidae) y diagnosis de formas nuevas ibero-marroquíes. Tamuda 2: 318-324.

Cobos A. 1969. Nuevos materiales para el estudio del Género Gynandrophthalma Lac. Graellsia 24: 67-77.

Codina Padilla F. 1961a. Apuntes sobre Chrysomelidae ibero-marroquíes (Coleoptera). Graellsia 19: 45-57.

Codina Padilla F. 1961b. Nota sobre las Chrysomela ibero-marroquies de subgenero Polysticta Hope (Col. Chrys.). Bull. Soc. Sci. Nat. Maroc 41: 69-83.

Colwell R.K. 2000. EstimateS: statistical estimation of species richness and shared species from samples. Version 6. User's guide and application published at: http://viceroy.eeb.uconn.edu/ estimates.

Colwell R.K. and Coddington J.A. 1994. Estimating terrestrial biodiversity through extrapolation. Philos. Trans. R. Soc. London B 345: 101-118.

Daccordi M. and Petitpierre E. 1977. Coleópteros Crisomélidos de la Sierra de Cazorla (Jaén) y descripción de una nueva especie de Clytra Laich. (Coleoptera, Chrysomelidae). Misc. Zool. 4(1): 225-236.

Doguet S., Bastazo G., Bergeal M. and Vela J.M. 1996. Contribution a l'étude des Chrysomelidae d'Andalousie (Coleoptera). Nouvelle Rev. d'Entomol. (N.S.) 13(4): 315-323. 
Fairmaire L. 1884. Liste complementaire des espéces du G. Timarcha. An. R. Soc. Esp. Hist. Nat. 13: $75-112$.

Franz H. 1958. Neue und ungenugend bekannte Coleopterenarten aus Spanien. Eos 34: 117-130. García-Ocejo A. and Gurrea P. 1995. Los crisomélidos (Coleoptera: Chrysomelidae) de la Sierra de Guadarrama (España Central). Análisis biogeográfico. Bol. Asoc. Española Entomol. 19(3-4): 51-68.

García-Ocejo A., Gurrea P. and Petitpierre E. 1992. Chrysomelidae (Coleoptera) de la sierra de Gredos (Sistema Central): datos faunísticos, ecológicos y fenológicos. Misc. Zool. 16: 81-92.

González de Andrés C. 1934. Insectos perjudiciales a las plantas cultivadas en Galicia. An. Asoc. Española Prog. Ciencias 1(4): 829-837.

Heyden L. von 1870. Entomologische Reise nach dem Sudlichen Spanien, der Sierra Guadarrama und Sierra Morena, Portugal und den Cantabrischen Gebirgen. Nicolaische Verlagbuckhandlung, Berlin.

Hortal J., García-Pereira P. and García-Barros E. 2004. Butterfly species richness in mainland Portugal: predictive models of geographic distribution patterns. Ecography 27: 68-82.

Hortal J., Lobo J.M. and Martín-Piera F. 2001. Forecasting insect species richness scores in poorly surveyed territories: the case of the Portuguese dung beetles (Col. Scarabaeinae). Biodiv. Conserv. 10: 1343-1367.

Iglesias L. 1928. Notas entomolóxicas. Insectos Galiza 1(53): 89-94.

Izco J. 1987. Galicia. In: Peinado-Lorca M. and Rivas-Martínez S. (eds), La vegetación de España. Universidad de Alcalá de Henares, Alcalá de Henares.

Jolivet P. 1953. Les Chrysomeloidea des Îles Baléars. Mém. Inst. R. Sci. Nat. Belg. 2(58): 3-152.

Král J. 1966. Zur Kenntnis der palaearktischen Altica-Arten. IV. Entomol. Blätter 62: 159-168.

Laboissière V. 1934. Galerucinae de la faune française (Coléoptères). Ann. Soc. Entomol. Fr. 103: $1-108$.

Leonardi C. and Doguet S. 1990. Studio critico sui Longitarsus del gruppo pratensis (Panzer) (Col. Chrysomelidae). Atti Soc. Ital. Sci. Nat. Museo Civico Storia Nat. 131(2): 13-74.

Lombardero J., Rodríguez-Fernandez R.J. and Fernández de Ana-Magan F.J. 1993. Altica quercetorum (Foudras, 1860) (Col. Chrys.) plaga de las "Carballeiras" gallegas. Actas I Congreso Forestal Español 3: 307-312.

López Seoane V. 1866. Reseña de la Historia Natural de Galicia. Imprenta de Soto Freire, Lugo. López Seoane V. 1877. Notas para la fauna gallega. Imprenta de El Eco Ferrolano, Ferrol.

Marseul S. 1883. Monographie de Chrysomelides de L'Ancien Monde. L'Abeille 21: 1-108.

Martín-Piera F. and Lobo J.M. 2000. Diagnóstico sobre el conocimiento sistemático y biogeográfico de tres órdenes de insectos hiperdiversos en España: Coleoptera, Hymenoptera y Lepidoptera. In: Martín-Piera F., Morrone J.J. and Melic A. (eds), Hacia un Proyecto CYTED para el Inventario y Estimación de la Diversidad Entomológica en Iberoamérica: prIBES-2000. Monografias Tercer Milenio, Vol. 1. SEA, Zaragoza, pp. 287-308.

Martín-Piera F. and Lobo J.M. 2003. Database records as a sampling effort surrogate to predict spatial distribution of insects in either poorly or unevenly surveyed areas. Acta Entomol. Ibérica Macaronésica 1: 23-35.

Medellín R.A. and Soberón J. 1999. Predictions of mammal diversity of four land masses. Conserv. Biol. 13: 143-149.

Meier R. and Dikow T. 2004. Significance of taxonomic databases from taxonomic revisions for estimating and mapping the global species diversity of invertebrates and repatriating reliable specimen data. Conserv. Biol. 18(2): 478-488.

Moreno C.E., and Halffter G. 2000. Assessing completeness of bat diversity inventories using species accumulation curves. J. Appl. Ecol. 37: 149-158.

Noguera F.A., Zaragoza-Caballero S., Chemsak J.A., Rodríguez-Palafox A., Ramírez E., González-Soriano E. and Ayala R. 2002. Diversity of the family Cerambycidae (Coleoptera) of the tropical dry forest of Mexico, I. Sierra de Huautla, Morelos. Ann. Entomol. Soc. Am. 95(5): $617-627$. 
Petersen F.T., Meier R. and Larsen M.N. 2003. Testing species richness estimation methods using museum label data on the Danish Asilidae. Biodiv. Conserv. 12: 687-701.

Petitpierre E. 1980. Catàleg dels Coleòpters Crisomèlids de Catalunya, I. Cryptocephalinae. Butll. Inst. Catalana d'Hist. Nat. (Sec. Zool.) 45: 65-76.

Petitpierre E. 1981. Chrysomelidae (Col.) de la Sierra de Albarracín. Bol. Asoc. Española Entomol. 4: 7-18.

Petitpierre E. 1983. Catàleg dels Coleòpters Crisomèlids de Catalunya, II. Zeugophorinae, Donaciinae, Criocerinae, Clytrinae, Lamprosomatinae i Eumolpinae. Butll. Inst. Catalana d'Hist. Nat. (Sec. Zool.) 49: 87-96.

Petitpierre E. 1988. Catàleg dels Coleòpters Crisomèlids de Catalunya, III. Chrysomelinae i Galerucinae. Butll. Inst. Cat. Hist. Nat. (Sec. Zool.) 55: 79-100.

Petitpierre E. 1994. Estudi faunístic i ecològic dels Coleòpters Crisomèlids de la Vall d'Aran. Butll. Inst. Catalana d'Hist. Nat. 62: $77-108$

Petitpierre E. 1997. Los Chrysomelidae (Coleoptera) del Moncayo (Aragón). Zapateri, Rev. Aragonesa Entomol. 7: 273-280.

Petitpierre E. 1999. Catàleg dels Coleòpters crisomèlids de Catalunya, IV. Alticinae. Butll. Inst. Catalana d'Hist. Nat. (Sec. Zool.) 67: 91-129.

Petitpierre E. 2000. Coleoptera, Chrysomelidae I. In: Ramos M.A. et al. (eds), Fauna Ibérica, Vol. 13. Museo Nacional de Ciencias Naturales, Madrid.

Petitpierre E. and Gómez-Zurita J. 1998. Los Chrysomelidae de León; NO de España (Coleoptera). Nouvelle Rev. d'Entomol. (N.S.) 15(1): 13-26.

Plaza Infante E. 1979. Contribución al conocimiento de los Cryptocephalus ibéricos. Bol. Asoc. Española Entomol. 3: 5-10.

Rivas-Martínez S. 1987. Memoria del Mapa de Series de Vegetación de España. Ministerio de Agricultura, Pesca y Alimentación, Madrid.

Soberón J. and Llorente J. 1993. The use of species accumulation functions for the prediction of species richness. Conserv. Biol. 7: 480-488.

Soberón J.M., Llorente J.B. and Oñate L. 2000. The use of specimen-label databases for conservation purposes: an example using Mexican Papilionid and Pierid butterflies. Biodiv. Conserv. 9: 1441-1466.

StatSoft. 2001. STATISTICA 6.0 (data analysis software system). StatSoft, Inc., Tulsa, OK.

Summerville K.S., Metzler E.H. and Crist T.O. 2001. Diversity of Lepidoptera in Ohio Forests at local and regional scales: how heterogeneous is the fauna? Ann. Entomol. Soc. Am. 94(4): 583591

Vela J.M. and Bastazo G. 1999. Ecological and biogeographical aspects of the Andalusian Leaf Beetle endemisms. In: Cox M.L. (ed.), Advances in Chrysomelidae Biology 1. Backhuys Publishers, Leiden, pp. 137-158.

Vigna Taglianti A., Audisio P.A., Belfiore C., et al. 1992. Riflessione di gruppo sui corotipi fondamentali della fauna W-paleartica ed in particolare italiana. Biogeographia 16: 159-179.

Vives E. 2000. Listado preliminar de los crisomélidos de Aragón (Coleoptera: Chrysomelidae). Cat. entomofauna aragon. 21: 11-18.

Vives E. and González C.F. 1994. Coleópteros Crisomélidos de la colección Español Auqué (1 ${ }^{\mathrm{a}}$ parte). (Coleoptera: Chrysomelidae). Zapateri, Rev. Aragonesa Entomol. 4: 49-60.

Vives E. and González C.F. 1998. Coleópteros Crisomélidos de la colección Español Auqué (2a parte) (Coleoptera: Chrysomelidae). Zapateri, Rev. Aragonesa Entomol. 8: 171-178. 